\title{
Gestão de portfólio de projetos: contribuições e tendências da literatura
}

\section{Project portfolio management: trends and contributions of literature}

\author{
Marly Monteiro de Carvalho ${ }^{1}$ \\ Paula Vilas Boas Viveiros Lopes Lopes ${ }^{1}$ \\ Daniela Santana Lambert Marzagão'
}

\begin{abstract}
Resumo: A necessidade crescente de melhores resultados com recursos cada vez mais escassos requer que as empresas selecionem e invistam apenas nos projetos capazes de gerar vantagem competitiva. $\mathrm{O}$ fato de este processo de seleção envolver interesses e riscos, uma efetiva gestão de portfólio de projetos pode fornecer suporte de forma estruturada e organizada. Este trabalho tem como objetivo traçar um panorama da literatura acadêmica em gestão de portfólio de projetos, descrevendo tendências e os principais temas abordados. A abordagem metodológica utilizada foi de revisão bibliográfica, adotando técnicas de análise bibliométricas e de análise de conteúdo. As buscas foram realizadas na base científica de dados ISI Web of Knowledge e na base Scielo. A amostra inicial de análise foi composta por 85 artigos publicados entre 1994 e 2009, expandida para as referências citadas nesses artigos. A amostra foi analisada com relação à evolução das publicações e citações, identificação dos principais periódicos, autores e obras, bem como os temas principais abordados. Os resultados indicam um significativo aumento de artigos publicados e citações ao longo do período. Os modelos de gestão de portfólio identificados na literatura convergem para os seguintes processos-chave: propostas de projetos; direcionadores estratégicos; identificação; avaliação; seleção; priorização; alocação de recursos; e monitoramento \& controle. As ferramentas mais citadas nos estudos da amostra foram classificadas em três grupos: métodos financeiros, programação matemática e modelos estatísticos. Finalmente, os critérios para seleção dos portfólios que se destacaram foram: o potencial de mercado, viabilidade econômico-financeira e riscos/ incertezas.
\end{abstract}

Palavras-chave: Gestão de projetos. Gestão de portfólio. Estudo bibliométrico.

\begin{abstract}
The growing need for better results with increasingly scarce resources requires the companies to select and invest only in projects that generate competitive advantage. The fact that this selection process involves interests and risks, an effective project portfolio management can provide effective support. This paper aims to provide an overview of academic literature on project portfolio management describing trends and the key topics. The methodological approach chosen was a literature review, adopting bibliometric techniques and content analysis. The search was conducted in the ISI Web of Knlowledge and Scielo databases. The initial sample for analysis consisted of 85 articles published between 1994 and 2009 and included the cited references. The sample was analyzed according to the evolution of publications and citations and identification of key journals, authors, studies, and topics. The results indicate a significant increase in the volume of published papers and citations over the period of time studied. The portfolio management models identified in the literature converge on the following key processes: project proposals, strategic drivers, identification, evaluation, selection, prioritization, resource allocation, and monitoring \& control. The most cited tools in the studies in the sample were classified into three groups: financial methods, mathematical programming, and statistical models. Finally, the portfolio selection criteria that stood out were market potential, economic and financial feasibility, and risks and uncertainties.
\end{abstract}

Keywords: Project management. Portfolio management. Bibliometric study.

\section{Introdução}

A gestão do portfólio de projetos pode ser definida como uma série de modelos, procedimentos e processos que visam administrar um conjunto de projetos de forma sistêmica (CARVALHO; RABECHINI JUNIOR, 2008). Segundo o PMI

(PROJECT..., 2008), a gestão de portfólio estrutura e coordena os componentes do portfólio de projetos, com o intuito de atingir as metas das empresas. A importância deste tema se deve à necessidade de se conseguir, com recursos financeiros, humanos

\footnotetext{
${ }^{1}$ Laboratório de Gestão de Projetos, Departamento de Engenharia de Produção, Escola Politécnica, Universidade de São Paulo - USP, Av. Professor Almeida Prado, trav. 2, 128, CEP 05508-900, São Paulo, SP, Brasil, e-mail: marlymc@usp.br; aplopes10@usp.br; dslm0401@usp.br
}

Recebido em 25/5/2011 — Aceito em 14/11/2012

Suporte financeiro: CAPES e CNPq. 
e tecnológicos limitados, selecionar e focar os projetos que confiram maior vantagem competitiva, de acordo com a estratégia adotada pela organização (ROZENFELD et al., 2006).

O processo de gestão de portfólio envolve análise e revisão constante, ou seja, periodicamente os projetos precisam ser analisados quanto a sua evolução, resultados e viabilidade em serem mantidos no portfólio da empresa (COOPER; EDGETT; KLEINSCHMIDT, 1999). A decisão de manter ou não um projeto pode envolver riscos e conflitos organizacionais se não executada de forma estruturada e clara, com a participação efetiva de todos os envolvidos (GHASEMZADEH; ARCHER; IYOGUN, 1999).

Com o intuito de minimizar os riscos e aumentar as chances de sucesso na gestão de portfólio, algumas empresas utilizam modelos da literatura, os quais sugerem práticas e indicam premissas para uma boa execução do processo.

O objetivo desta pesquisa é conhecer o panorama da produção acadêmica sobre o tema gestão de portfólio de projetos, identificando os periódicos de destaque, autores que mais abordaram o tema e os trabalhos mais representativos. Para atender aos objetivos, optou-se por uma estratégia de revisão sistemática de literatura híbrida, mesclando análise bibliométrica e análise de conteúdo.

O trabalho está estruturado nas seções de metodologia de pesquisa, resultados e conclusões. Na seção 2, metodologia de pesquisa, poderá ser encontrado o detalhamento da forma de coleta e tratamento dos dados. Em seguida, na seção 3, serão apresentados os resultados obtidos sob o ponto de vista da análise bibliométrica por meio de indicadores de publicações e citações. Na seção 4 , serão apresentadas as discussões dos resultados pautadas na análise de conteúdo. Na seção 5 , serão destacadas as conclusões, sugestões para trabalhos futuros e as limitações da pesquisa.

\section{Metodologia de pesquisa}

A metodologia de pesquisa engloba estratégias de coleta de informação na realidade analisada pelo pesquisador (MEKSENAS, 2009). Conforme mencionado na seção introdutória, a abordagem metodológica selecionada foi a de revisão sistemática de literatura, que mesclou análise bibliométrica e análise de conteúdo. Para traçar o panorama da literatura acadêmica na área de gestão de portfólio de projetos, optou-se pela análise bibliométrica, que pode ser definida como uma série de técnicas que visam quantificar o processo da comunicação escrita (IKPAAHINDI, 1985).

A pesquisa bibliométrica visa responder se existem padrões na literatura pesquisada e permite identificar quais os periódicos que mais publicaram artigos sobre o tema, qual a evolução destas publicações ao longo do tempo e quais as áreas mais relacionadas à temática pesquisada, conforme sugerido por Prasad e Tata (2005). Por outro lado, para Neely (2005), a pesquisa bibliométrica analisa as citações, o que permite identificar os trabalhos que mais impactaram a área, bem como a relação entre os trabalhos e entre esses e suas referências mais citadas, por meio das redes de citação.

A análise quantitativa das citações de artigo pode ser realizada por meio da contagem do número de citações individuais de cada texto, bem como da análise das referências bibliográficas utilizadas pelos artigos mais citados.

A análise das referências bibliográficas identifica os fenômenos de agrupamento bibliométrico, bem como as relações significativas entre dois artigos com base no número de referências em comum (KESSLER, 1963). Optou-se por elaborar a rede de artigos para referência, pois ela fornece não só um panorama dos artigos mais citados na amostra, mas também de suas referências mais citadas, articulando os fundamentos teóricos da área.

Para melhor identificar os principais temas abordados, optou-se pela análise de conteúdo, de forma a completar a abordagem quantitativa da análise bibliométrica. Dessa forma, foi feita uma análise em profundidade dos estudos da amostra, seguindo os procedimentos de análise de conteúdo, sugeridos por diversos autores (WHITE; McCAIN, 1998; RAMOS-RODRIGUEZ; RUIZ-NAVARRO, 2004).

\subsection{Objetivos principais e secundários}

Este trabalho tem como objetivo principal traçar um panorama da literatura acadêmica em gestão de portfólio de projetos, descrevendo tendências e os principais temas abordados.

Os objetivos secundários, alinhados com a análise bibliométrica e de conteúdo são:

- Verificar os periódicos que constituem o principal fórum de discussão da temática de gestão de portfólio, publicando o maior número de artigos;

- Acompanhar os padrões de evolução das publicações ao longo dos anos estratificados por periódico e artigo (número de artigos publicados e citações);

- Identificar as principais áreas relacionadas com o tema;

- Identificar os autores e obras que mais impactaram a área, utilizando como proxy de impacto o número de citações;

- Analisar a relação entre trabalhos e referências mais citados, por meio das redes de citações; e

- Identificar os principais temas, tendências e lacunas na literatura. 


\subsection{Amostra e procedimento}

O critério de escolha dos artigos da amostra inicial foi a combinação dos tópicos "portfolio management" e "project management" na base de dados ISI Web of Knlowledge (Web of Science) e "portfolio management" na base de dados Scielo. A busca foi realizada sem especificar filtro por área, periódico ou restrições temporais. Optou-se por analisar apenas artigos na amostra inicial, pelo fato de passarem por processo de avaliação por pares e por conterem as informações necessárias para a análise bibliométrica, tais como autores, referências, número de citações, periódico, ano de publicação, etc.

A busca resultou inicialmente em 86 artigos na base de dados ISI Web of Knowledge (entre $1994 \mathrm{e}$ 2010) e 10 na base Scielo (entre 2003 e 2010). A Figura 1 mostra o fluxo de trabalho realizado com estes 96 artigos.

Depois da leitura dos resumos, oito artigos (9\%), da base ISI Web of Knowledge (Web of Science), foram excluídos e três (10\%), da base Scielo, por se tratarem de trabalhos mais voltados a portfólio de investimentos, não se enquadrando à temática desta pesquisa; resultando, na amostra, 85 trabalhos, os quais foram lidos completamente. A fim de assegurar maior confiabilidade neste rastreio inicial, os pesquisadores leram separadamente os resumos, e apenas os artigos que foram unanimemente considerados como estando fora do âmbito da pesquisa foram eliminados. Para a construção das redes de citação, os dados foram tratados no software Sitkis - versão 2.0 (SCHILDT, 2002) e as redes foram desenhadas com o auxílio do software Ucinet 6 - versão 6.289 (BORGATTI; EVERETT; FREEMAN, 2002).

\subsection{Análise I - Indicadores de publicações}

Os 85 artigos foram codificados em áreas primárias e secundárias, ou seja, foram identificadas as duas áreas mais relacionadas com cada um dos artigos, conforme sugerido por Prasad e Tata (2005). A primeira análise foi a de publicações por periódico e ano, que identificou os periódicos de maior destaque, bem como a evolução das publicações ao longo do tempo. Em seguida, analisaram-se as publicações por área e ano, caracterizando a existência das áreas mais presentes, identificando possíveis aglomerados. Por fim, verificou-se a tabulação cruzada das áreas. Posteriormente, os artigos foram analisados em profundidade.

\subsection{Análise II - Indicadores de citações}

A primeira análise de citações foi a dos artigos mais citados, a qual identificou trabalhos representativos no tema gestão de portfólio, bem como a corrente de pesquisa dos seus respectivos autores.

A segunda análise foi a de citações de artigos para referências, que permitiu resgatar trabalhos representativos que não fizeram parte da amostra, tais como trabalhos de outras bases de dados, bem como livros e proceedings, uma vez que esta rede visualiza não só os trabalhos da amostra, como também todas as referências citadas por eles. Por último, foi construída a rede de colaboração entre países, que possibilitou a análise de parcerias na produção acadêmica dos trabalhos da amostra.

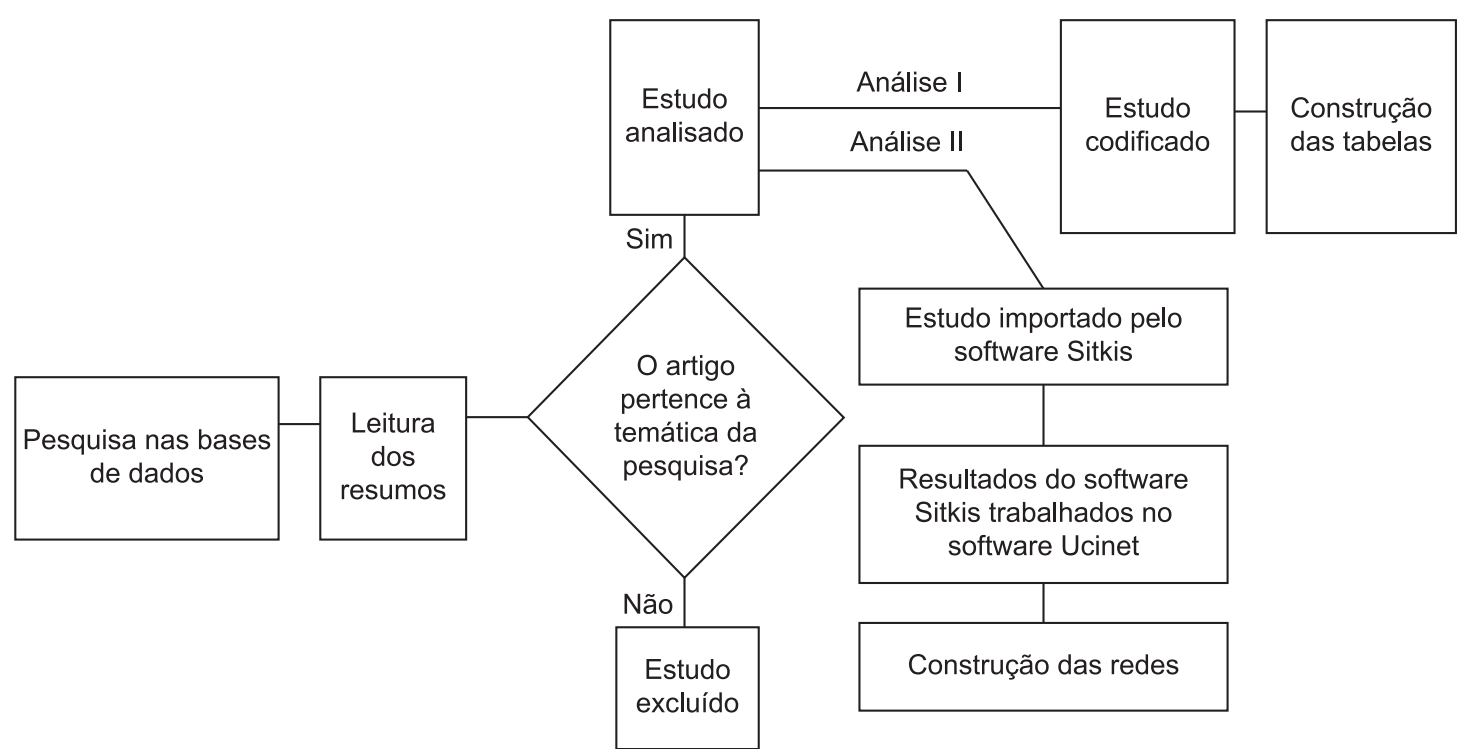

Figura 1. Fluxo de trabalho do estudo bibliométrico. 


\section{Resultados da pesquisa}

As seções seguintes apresentam as principais descobertas desta pesquisa.

\subsection{Análise I - Indicadores de publicações}

A Tabela 1 mostra o número de publicações por periódico, no período entre 1994 e 2009. Os 85 artigos foram publicados em 51 periódicos de áreas como gestão, engenharia, tecnologia, ciências sociais, educação, pesquisa operacional e negócio. A média de publicações foi de 1,7 artigos por periódico, o que reforça a informação de multidisciplinaridade de áreas do tema gestão de portfólio.

Aproximadamente 33\% dos artigos foram publicados em seis periódicos, os quais publicaram pelo menos três artigos, sendo eles:

- Research Technology Management: periódico de inovação tecnológica, com foco em pesquisas e desenvolvimento de novos produtos;

- Journal of Product Innovation Management: periódico que visa unir a teoria acadêmica de inovação com a prática efetiva de gerenciamento;

- Drug Information Journal: periódico dedicado a divulgar informações para a pesquisa de medicamentos, promovendo a comunicação entre educação, pesquisa, indústria e governo;

- IEEE Transactions on Engineering Management: um dos periódicos da IEEE, uma organização sem fins lucrativos, líder mundial em associação profissional para o avanço da tecnologia em áreas como sistemas aeroespaciais, computadores, telecomunicação e engenharia biomédica; e

- DYNA: periódico com foco em pesquisas de engenharia industrial.

Constatou-se um aumento quase que linear das publicações, com um pico no ano de 2010. Na Figura 2, é possível verificar a rede de palavras-chave. Em todas as redes desta pesquisa, foi realizado um filtro para limitar o número de "nós", com o intuito de apresentá-las de forma mais clara. O guia do usuário do software Sitkis sugere incluir referências que são citadas em, pelo menos, $1 \%-10 \%$ de todos os artigos.

A Tabela 2 mostra os índices de centralidade das palavras-chave, os quais indicam as mais relacionadas com as demais. Analisando a relação das áreas temáticas com as palavras-chave de busca deste trabalho, "portfolio management" e "project management", com todas as demais citadas pelos autores, foi possível identificar áreas mais fortemente ligadas ao tema, as quais são apresentadas resumidamente a seguir, de acordo com a forma como apareceram nos trabalhos analisados:

- Performance / Desempenho (A1): uma das áreas campeãs deste estudo. $\mathrm{O}$ aumento da eficiência é, sem dúvida, um dos principais objetivos daqueles que decidem se dedicar à gestão de portfólio. Como obter melhores resultados, maior desempenho e crescimento constante;

- Product Development / Desenvolvimento de Produto (A2): principalmente de pesquisa tecnológica e de inovação de produto. Um bom desenvolvimento de produto pode não só gerar ganhos financeiros, como também evitar desperdícios e permitir, à empresa, melhor posicionamento em seu setor;

- Research and Development / Pesquisa \& Desenvolvimento (A3): uma área bastante presente, principalmente em pesquisas médicas, químicas, de engenharia e tecnológicas; e

- Risk and Uncertainty / Risco e Incerteza (A4): outra área muito explorada neste estudo. A grande maioria das empresas que buscam a gestão de portfólio busca minimizar riscos de investimentos inadequados, escolha equivocada de projetos, má alocação de recursos humanos.

A Tabela 3 mostra a classificação dos artigos por periódico e área. É importante observar que alguns dos temas estiveram mais relacionados a determinado perfil de periódico, o que era esperado, dada a política editorial praticada. A área que mais se destacou na amostra foi Pesquisa \& Desenvolvimento, seguida por Desenvolvimento de Produto, Desempenho e Risco e Incerteza.

A Tabela 4 apresenta a intersecção entre as áreas primária e secundária.

Observa-se que a área de Desempenho foi a que se relacionou com maior frequência com as demais áreas. Vale também notar um forte relacionamento existente entre risco e pesquisa $\&$ desenvolvimento.

\subsection{Análise II - Indicadores de citações}

A primeira análise de citações foi a de artigos mais citados. Utilizou-se, como critério de corte, o grupo de trabalhos que foram citados mais do que dez vezes. Com base nesse critério, obteve-se a relação de 20 artigos, descrita na Tabela 5.

Para estes trabalhos, foi realizada uma análise mais qualitativa, identificando os objetivos de pesquisa e algumas das principais conclusões encontradas pelos autores. Vale destacar que para um total de 530 citações, dos 85 artigos no período analisado, o grupo de artigos mais citados representa 449 citações $(\sim 85 \%)$. Nesse grupo de artigos, observa-se o grau de centralidade de Cooper, Edgett e Kleinschmidt (1999), que representa 19\% do total de citações.

A Figura 3 apresenta a evolução do número de citações ao longo do período de análise, para o total do grupo de artigos mais citados, bem como discriminada 


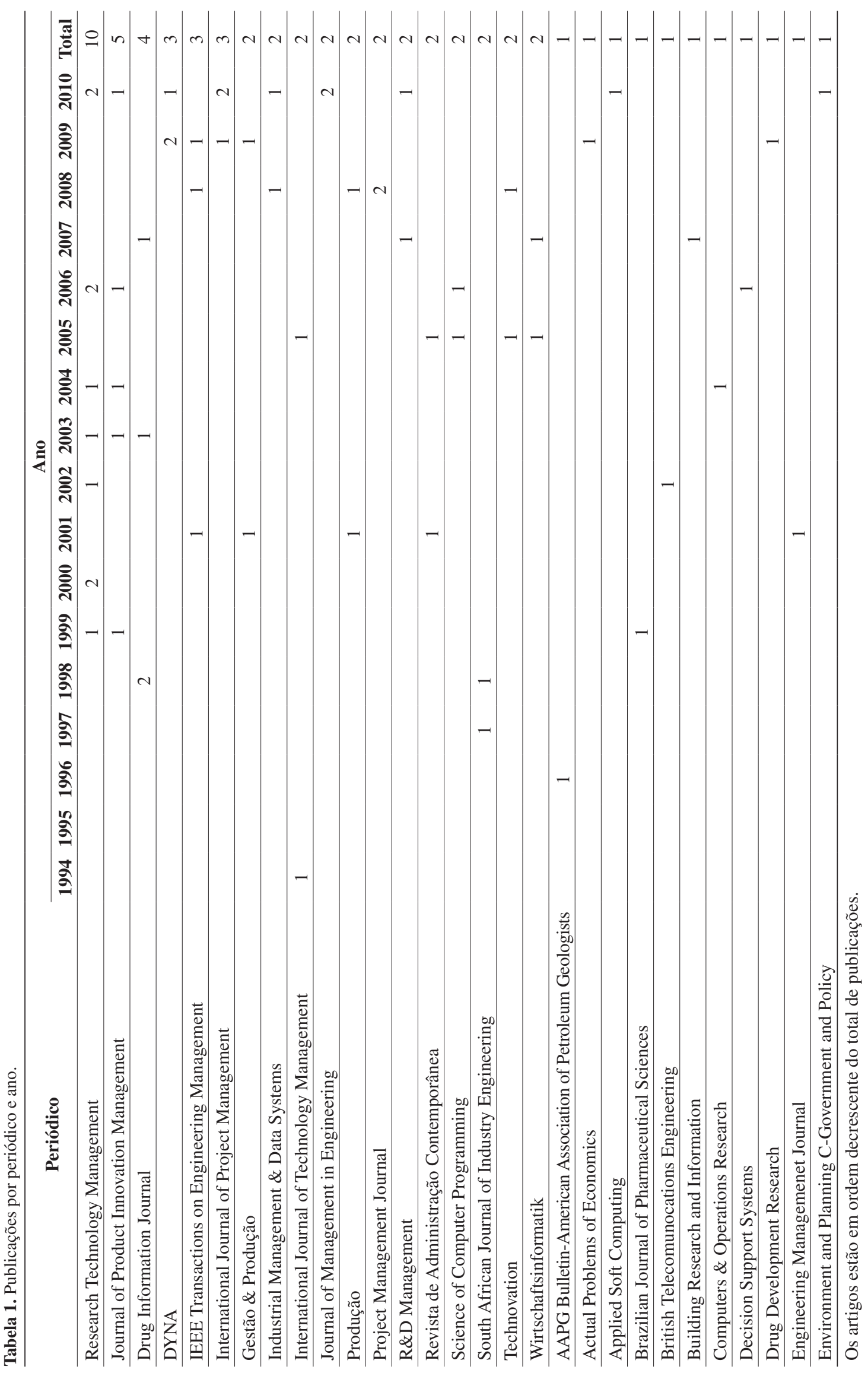




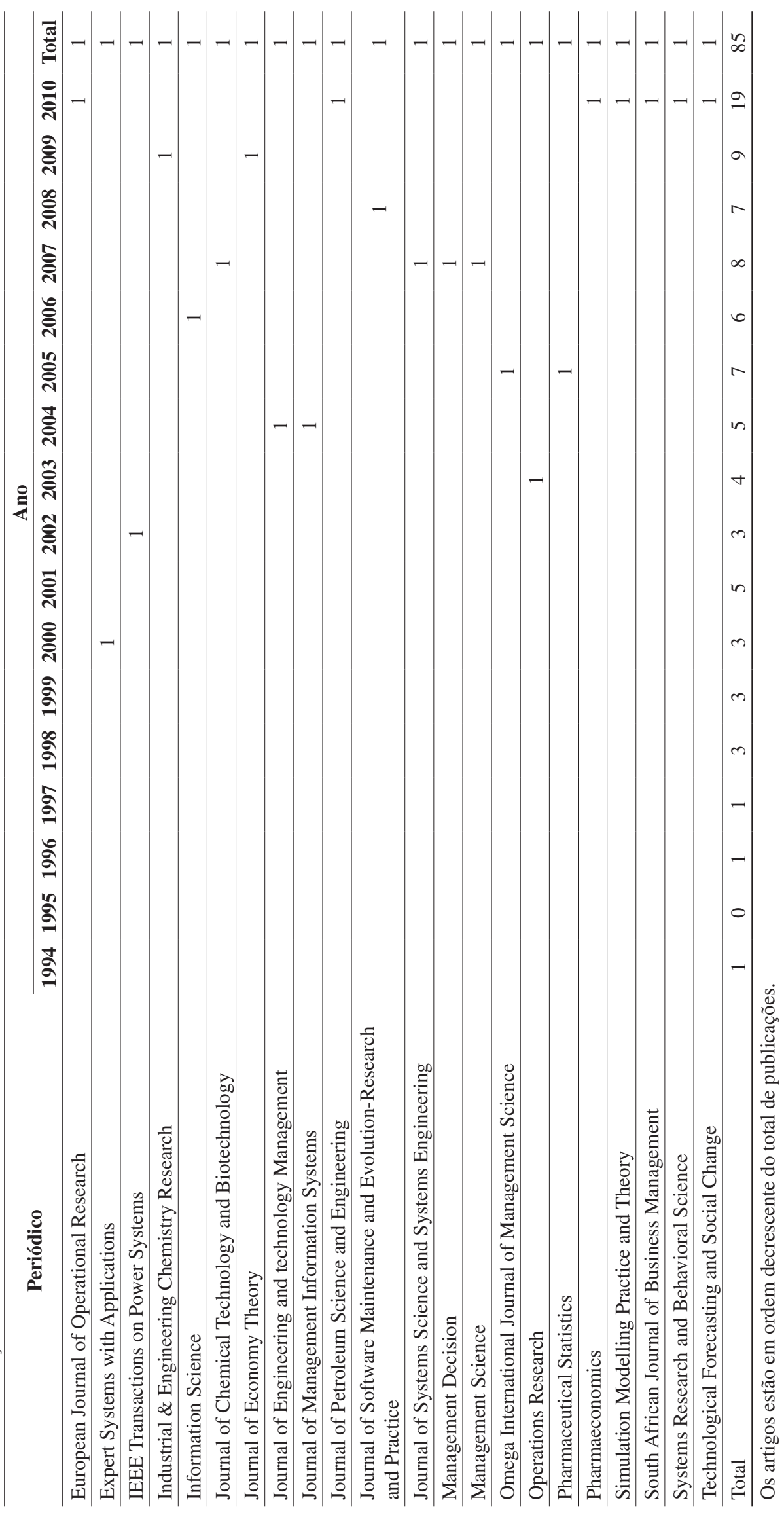




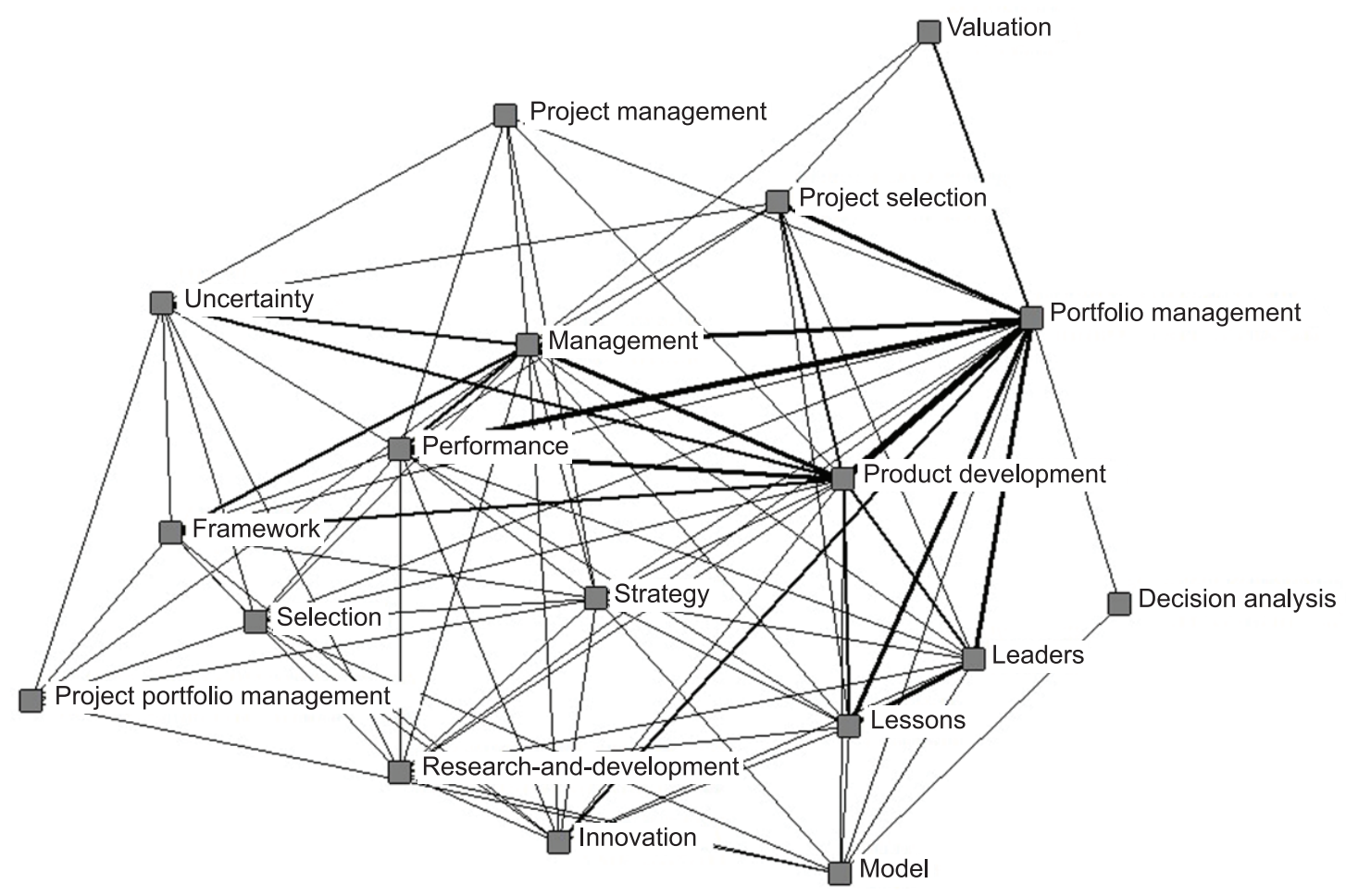

Figura 2. Rede de palavras-chave. As espessuras das linhas representam a intensidade das relações.

Tabela 2. Índices de centralidade da rede de palavras-chave.

\begin{tabular}{lclc}
\hline \multicolumn{1}{c}{ Palavras-chave } & Índices de centralidade & \multicolumn{1}{c}{ Palavras-chave } & Índices de centralidade \\
\hline Portfolio management & 54.000 & Innovation & 15.000 \\
Product development & 41.000 & Selection & 14.000 \\
Management & 31.000 & Uncertainty & 13.000 \\
Performance & 29.000 & Research and development & 12.000 \\
Lessons & 19.000 & Project management & 11.000 \\
Leaders & 19.000 & Model & 10.000 \\
Strategy & 18.000 & Project portfolio management & 8.000 \\
Framework & 17.000 & Valuation & 6.000 \\
Project selection & 15.000 & Decision analysis & 5.000 \\
$\quad$ Estatísticas Descritivas & & & \\
Média & 18.722 & & \\
Soma & 337.000 & & \\
Mínimo & 5.000 & & \\
Máximo & 54.000 & & \\
Número de nós & 18 & & \\
\hline As palacha & &
\end{tabular}

As palavras-chave estão apresentadas em ordem decrescente do índice de centralidade.

por trabalho. Observando-se a Figura 4, pode-se notar que existem poucas citações destes trabalhos até 2002. O crescimento das citações se deu a partir de 2003, com picos em 2006, 2008 e 2010, indicando uma área de conhecimento em crescimento.

Num primeiro momento, entre 2000 e 2001, receberam citações os trabalhos: Cooper, Edgett e Kleinschmidt (1999), cujos resultados mostraram que empresas com boa gestão de portfólio possuem projetos com melhor desempenho e mais alinhados com a estratégia da companhia; Siddiqi (2000), no qual o autor concluiu que os métodos desenvolvidos na pesquisa tiveram aplicabilidade dentro do mercado de Energia por tratar de maneira estocástica o comportamento de demandas, preços de energia e seus respectivos riscos.

Num segundo momento, entre 2002 e 2004, também foram citados os artigos: Cooper, Edgett 
Tabela 3. Publicações por periódico e área.

\begin{tabular}{|c|c|c|c|c|c|}
\hline \multirow{2}{*}{ Periódico } & \multicolumn{5}{|c|}{ Área } \\
\hline & A1 & A2 & $\mathbf{A 3}$ & A4 & Total \\
\hline Research Technology Management & 3 & 2 & 3 & 2 & 10 \\
\hline Journal of Product Innovation Management & & 5 & & & 5 \\
\hline Drug Information Journal & 1 & & 3 & & 4 \\
\hline DYNA & & 1 & 2 & & 3 \\
\hline IEEE Transactions on Engineering Management & 1 & 1 & 1 & & 3 \\
\hline International Journal of Project Management & 1 & 1 & 1 & & 3 \\
\hline Gestão \& Produção & & 1 & & 1 & 2 \\
\hline Industrial Management \& Data Systems & 1 & & 1 & & 2 \\
\hline International Journal of Technology Management & 2 & & & & 2 \\
\hline Journal of Management in Engineering & 1 & 1 & & & 2 \\
\hline Produção & 2 & & & & 2 \\
\hline Project Management Journal & & & 1 & 1 & 2 \\
\hline R\&D Management & & 1 & & 1 & 2 \\
\hline Revista de Administração Contemporânea & 1 & 1 & & & 2 \\
\hline Science of Computer Programming & 1 & 1 & & & 2 \\
\hline South African Journal of Industry Engineering & & & 2 & & 2 \\
\hline Technovation & 2 & & & & 2 \\
\hline Wirtschaftsinformatik & 1 & & & 1 & 2 \\
\hline AAPG Bulletin-American Association of Petroleum Geologists & & & & 1 & 1 \\
\hline Actual Problems of Economics & & & & 1 & 1 \\
\hline Applied Soft Computing & & 1 & & & 1 \\
\hline Brazilian Journal of Pharmaceutical Sciences & & 1 & & & 1 \\
\hline British Telecomunocations Engineering & & & 1 & & 1 \\
\hline Building Research and Information & 1 & & & & 1 \\
\hline Computers \& Operations Research & & & & 1 & 1 \\
\hline Decision Support Systems & & & 1 & & 1 \\
\hline Drug Development Research & & & 1 & & 1 \\
\hline Engineering Managemenet Journal & 1 & & & & 1 \\
\hline Environment and Planning C-Government and Policy & & & 1 & & 1 \\
\hline European Journal of Operational Research & & & & 1 & 1 \\
\hline Expert Systems with Applications & & 1 & & & 1 \\
\hline IEEE Transactions on Power Systems & 1 & & & & 1 \\
\hline Industrial \& Engineering Chemistry Research & & & 1 & & 1 \\
\hline Information Science & & & 1 & & 1 \\
\hline Journal of Chemical Technology and Biotechnology & & & 1 & & 1 \\
\hline Journal of Economy Theory & & & & 1 & 1 \\
\hline Journal of Engineering and technology Management & & 1 & & & 1 \\
\hline Journal of Management Information Systems & & 1 & & & 1 \\
\hline Journal of Petroleum Science and Engineering & 1 & & & & 1 \\
\hline Journal of Software Maintenance and Evolution-Research and Practice & & & & 1 & 1 \\
\hline Journal of Systems Science and Systems Engineering & & & & 1 & 1 \\
\hline Management Decision & & 1 & & & 1 \\
\hline Management Science & & & 1 & & 1 \\
\hline Omega International Journal of Management Science & & & 1 & & 1 \\
\hline Operations Research & & & & 1 & 1 \\
\hline Pharmaceutical Statistics & & 1 & & & 1 \\
\hline Pharmaeconomics & & & 1 & & 1 \\
\hline Simulation Modelling Practice and Theory & & 1 & & & 1 \\
\hline South African Journal of Business Management & 1 & & & & 1 \\
\hline Systems Research and Behavioral Science & & & 1 & & 1 \\
\hline Technological Forecasting and Social Change & & 1 & & & 1 \\
\hline Total & 22 & 24 & 25 & 14 & 85 \\
\hline
\end{tabular}

Os artigos estão em ordem decrescente do total de publicações. 
Tabela 4. Tabulação cruzada da área primária com a secundária.

\begin{tabular}{ccccc}
\hline \multirow{2}{*}{ Área primária } & \multicolumn{4}{c}{ Área secundária } \\
\cline { 2 - 5 } & TA1 & TA2 & TA3 & TA4 \\
\hline TA1 & - & 11 & 4 & 6 \\
TA2 & 20 & - & 2 & 2 \\
TA3 & 12 & 5 & - & 8 \\
TA4 & 9 & 3 & 3 & - \\
\hline
\end{tabular}

e Kleinschmidt (2000), cuja pesquisa indicou que os principais motivos que podem levar a um baixo desempenho do gerenciamento de projetos são: muitos projetos para poucos recursos, informações imprecisas, muitos projetos pequenos; Mikkola (2001), no qual o autor argumentou sobre a importância do tratamento do portfólio como instrumento estratégico para o posicionamento da empresa em termos de crescimento e sustentabilidade dos resultados; Dickinson, Thornton e Graves (2001), cujo modelo apresentado gerou

Tabela 5. Trabalhos mais citados.

\begin{tabular}{|c|c|c|}
\hline Artigo & Citações & Objetivos \\
\hline $\begin{array}{l}\text { Bardhan, Bagchi e } \\
\text { Sougstad (2004) }\end{array}$ & 22 & $\begin{array}{l}\text { Desenvolver um modelo de opções reais para priorização de projetos de } \\
\text { tecnologia da informação. }\end{array}$ \\
\hline Blau et al. (2004) & 27 & $\begin{array}{l}\text { Estabelecer um método para a seleção de sequências de projetos para a } \\
\text { indústria farmacêutica. }\end{array}$ \\
\hline Coldrick et al. (2005) & 20 & $\begin{array}{l}\text { Analisar a evolução e o uso de ferramentas na seleção de projetos, testando } \\
\text { um modelo. }\end{array}$ \\
\hline Cooper e Edgett (2003) & 13 & $\begin{array}{l}\text { Compreender os motivos geradores da falta de recursos humanos e de tempo } \\
\text { em projetos de desenvolvimento de novos produtos. }\end{array}$ \\
\hline $\begin{array}{c}\text { Cooper, Edgett e } \\
\text { Kleinschmidt (1999) }\end{array}$ & 87 & $\begin{array}{l}\text { Obter insights sobre quais métodos de gestão de portfólio são utilizados pelas } \\
\text { empresas. }\end{array}$ \\
\hline $\begin{array}{c}\text { Cooper, Edgett e } \\
\text { Kleinschmidt (2000) }\end{array}$ & 14 & $\begin{array}{l}\text { Descobrir os principais motivos geradores de baixa eficiência no gerenciamento } \\
\text { de projetos de algumas empresas. }\end{array}$ \\
\hline $\begin{array}{c}\text { Cooper, Edgett e } \\
\text { Kleinschmidt (2002b) }\end{array}$ & 10 & Identificar práticas eficientes de gerenciamento de projetos. \\
\hline $\begin{array}{l}\text { Cooper, Edgett e } \\
\text { Kleinschmidt (2004b) }\end{array}$ & 29 & $\begin{array}{l}\text { Identificar as iniciativas tomadas pelas empresas com ótimo desempenho em } \\
\text { desenvolvimento de novos produtos para transformar diretrizes estratégicas } \\
\text { em projetos. }\end{array}$ \\
\hline $\begin{array}{l}\text { Dickinson, Thornton e } \\
\text { Graves (2001) }\end{array}$ & 28 & $\begin{array}{l}\text { Apresentar um modelo desenvolvido por uma companhia americana, o qual } \\
\text { visa otimizar a carteira de projetos de desenvolvimento de novos produtos. }\end{array}$ \\
\hline $\begin{array}{l}\text { Girotra, Terwiesch e } \\
\text { Ulrich (2007) }\end{array}$ & 12 & Estabelecer um método para a avaliação da carteira de projetos. \\
\hline Gustafsson e Salo (2005) & 12 & $\begin{array}{l}\text { Desenvolver um modelo de gestão de portfólio considerando incertezas } \\
\text { exógenas, modelos de recursos por meio de variáveis dinâmicas e perfil de } \\
\text { risco da empresa. }\end{array}$ \\
\hline Hart et al. (2003) & 27 & $\begin{array}{l}\text { Identificar os critérios utilizados em cada fase do desenvolvimento de novos } \\
\text { produtos inovadores. }\end{array}$ \\
\hline Lin e Hsied (2004) & 19 & $\begin{array}{l}\text { Desenvolver um framework, com base no conceito de sistema de apoio à } \\
\text { decisão (decision support system). }\end{array}$ \\
\hline Lint e Pennings (2001) & 10 & $\begin{array}{l}\text { Demonstrar um modelo de uso do modelo de real options para suportar as } \\
\text { decisões de continuidade de projetos de desenvolvimento de novos produtos. }\end{array}$ \\
\hline Mikkola (2001) & 19 & $\begin{array}{l}\text { Demonstrar o uso da matriz de portfólio de pesquisa e desenvolvimento na } \\
\text { análise de projetos. }\end{array}$ \\
\hline Miller (2005) & 11 & $\begin{array}{l}\text { Propor análises do início do processo de desenvolvimento de novos produtos } \\
\text { da indústria farmacêutica. }\end{array}$ \\
\hline Mojsilović et al. (2007) & 10 & $\begin{array}{l}\text { Apresentar uma metodologia estatística de análise para o gerenciamento de } \\
\text { projetos de outsourcing. }\end{array}$ \\
\hline $\begin{array}{l}\text { Rogers, Gupta e } \\
\text { Maranas (2002) }\end{array}$ & 23 & $\begin{array}{l}\text { Apresentar um modelo otimizado para tomada de decisão na escolha de } \\
\text { projetos. }\end{array}$ \\
\hline Siddiqi (2000) & 22 & $\begin{array}{l}\text { Determinar a aplicabilidade da teoria de precificação de opções e análise de } \\
\text { decisões para a avaliação de projetos de investimento em geração de energia. }\end{array}$ \\
\hline Wang e Hwang (2007) & 34 & $\begin{array}{l}\text { Apresentar um modelo para diminuir o impacto da incerteza nos portfólios de } \\
\text { projetos. }\end{array}$ \\
\hline
\end{tabular}

Os artigos estão em ordem decrescente do total de citações. 
benefícios, tais como minimização do tempo de reavaliação do portfólio e balanceamento de carteira.

Num terceiro momento, entre 2005 e 2007, passaram a ser citados os artigos: Lint e Pennings (2001), no qual os autores concluíram que é importante para o portfólio manter um método único e transparente para a comparação de produtos em diferentes estágios do desenvolvimento; Cooper, Edgett e Kleinschmidt (2002b), no qual identificaram que as empresas com melhores práticas de gestão de portfólio utilizam o modelo "stage-gates"; Miller (2005), no qual percebeu que a quantidade de dados obtida em pesquisa farmacêutica não garante melhores condições

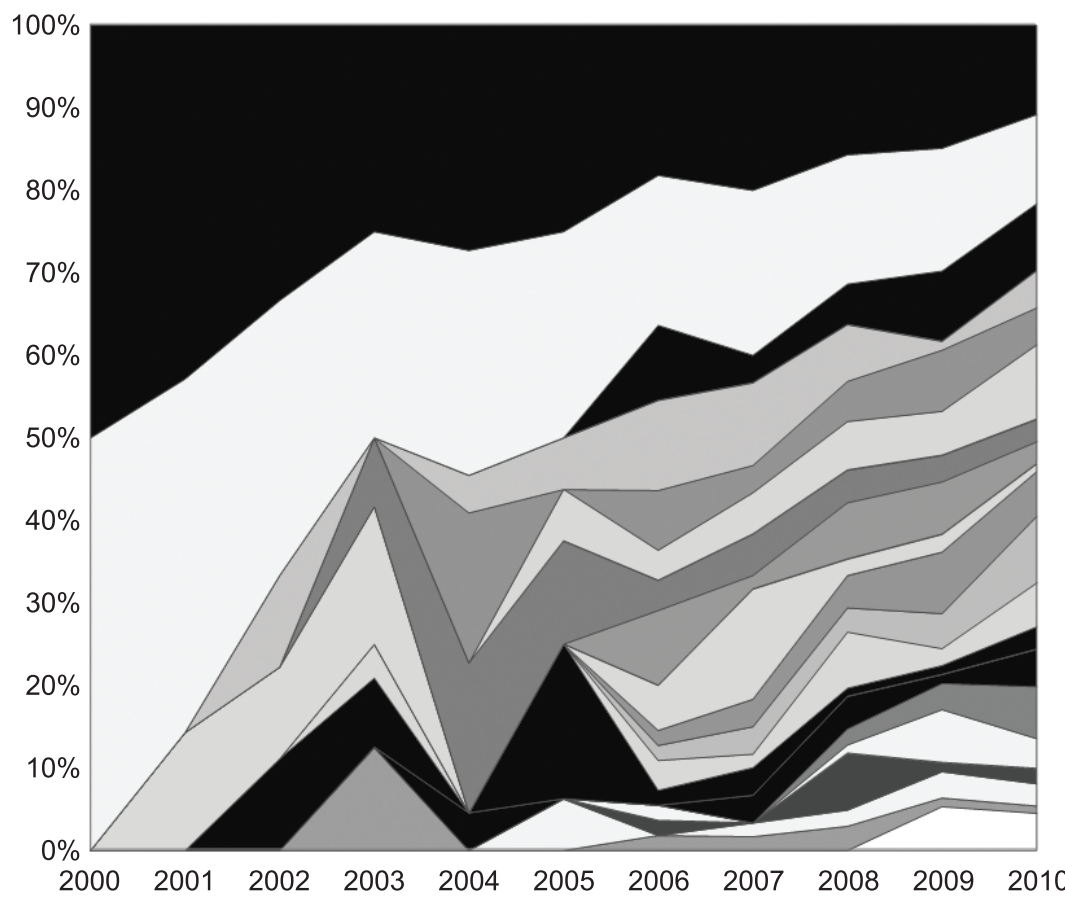

- Cooper, Edgett e Kleinschmidt (1999)

$\square$ Wang e Hwang (2007)

- Cooper, Edgett e Kleinschmidt (2004b)

$\square$ Dickinson, Thornton e Graves (2001)

a Hart et al. (2003)

$\square$ Blau et al. (2004)

- Rogers, Gupta e Maranas (2002)

- Bardhan, Bagchi e Sougstad (2004)

$\square$ Siddiqi (2000)

- Coldrick et al. (2005)

$\square$ Lin e Hsied (2004)

$\square$ Mikkola (2001)

- Cooper, Edgett e Kleinschmidt (2000)

- Cooper e Edgett (2003)

- Girotra, Terwiesch e Ulrich (2007)

$\square$ Gustafsson e Salo (2005)

- Miller (2005)

$\square$ Cooper, Edgett e Kleinschmidt (2002b)

• Lint e Pennings (2001)

$\square$ Mojsilović et al. (2007)

Figura 3. Evolução das citações dos artigos.

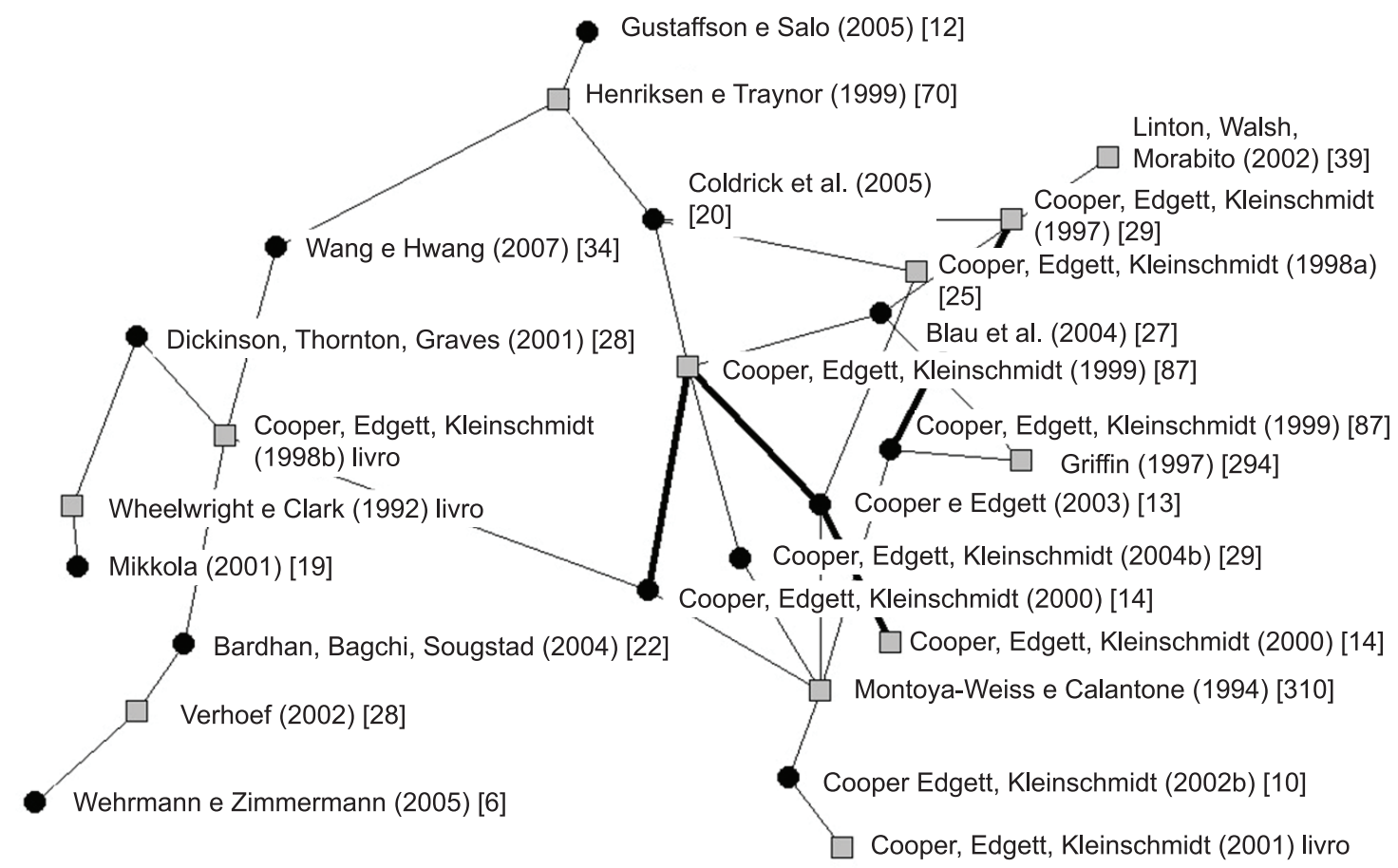

Figura 4. Rede de citação de artigos para referências. As espessuras das linhas representam a intensidade das relações. As informações entre parênteses representam os anos, e as entre colchetes o número de citações recebidas pelo artigo. 
na tomada de decisões; Gustafsson e Salo (2005), cujo framework apresentado pôde ser utilizado para a análise de problemas de investimentos de projetos; Cooper e Edgett (2003), em que notaram que o problema de escassez de recursos é gerado principalmente pela preocupação, por parte das empresas, em se desenvolverem no curto prazo, ignorando o acompanhamento e análise dos projetos, bem como a morte daqueles que, de alguma forma, não são mais viáveis; Lin e Hsied (2004), cujos principais benefícios do modelo, encontrados no estudo foram: facilidade de utilização e envolvimento de todos; Coldrick et al. (2005), em que identificaram, como benefício do uso do modelo de seleção, a estrutura formal de decisão e comunicação de informações sobre projetos; Bardhan, Bagchi e Sougstad (2004), cujo modelo permitiu analisar o valor e a relevância de priorizar projetos de tecnologia da informação, além de ser possível observar as interdependências entre projetos candidatos que aumentam muito a complexidade e desafios do modelo de priorização; Rogers, Gupta e Maranas (2002), cujos resultados mostraram que a chance de abandono de um projeto aumenta de acordo com as incertezas do mercado e probabilidades de insucesso dos ensaios clínicos; Hart et al. (2003), no qual concluíram que métricas relativas à unicidade do produto, potencial de mercado e viabilidade técnica estão concentrados nas fases iniciais do desenvolvimento; Blau et al. (2004), no qual concluíram que o método proposto, embora trabalhoso, proporcionou uma visão mais profunda aos gestores sobre as decisões do portfólio através do tempo; Cooper, Edgett e Kleinschmidt (2004b), que indicaram que as empresas com melhor desempenho tiveram foco estratégico em desenvolvimento de novos produtos.

Num período mais recente, entre 2008 e 2010, também receberam citações artigos de: Mojsilović et al. (2007), cujos resultados mostraram que diferentes fatores podem afetar as decisões em projetos de outsourcing e que o conhecimento destes fatores pode ser importante para os clientes e fornecedores no momento da escolha dos projetos; Girotra, Terwiesch e Ulrich (2007), no qual foi evidenciado que o valor de mercado de uma empresa reduz quando é publicada a notícia de uma falha em um projeto de desenvolvimento de novos produtos; Wang e Hwang (2007), cujo modelo desenvolvido contribuiu para a seleção de portfólios de projetos dentro de um cenário de ausência ou insuficiência de informação confiável para a minimização de riscos.

Outra análise foi a de artigos para referências (ver Figura 4), em que os círculos são os artigos e os quadrados as referências.

Foram resgatados, nesta rede, dois clássicos da literatura, o livro de Wheelwright e Clark (1992), que abordou o desenvolvimento de produto de forma revolucionária; e o de Cooper, Edgett e Kleinschmidt (1998b, 2001), que focou a gestão de portfólio para desenvolvimento de produto (trata-se do mesmo livro, porém foram citadas edições diferentes). Apareceram também na rede artigos clássicos, tais como o de Montoya-Weiss e Calantone (1994), que abordaram fatores que podem favorecer o desenvolvimento de produto; e de Griffin (1997), cuja autora apresentou práticas de desenvolvimento de produtos. Nota-se que as relações mais intensas aconteceram entre alguns dos trabalhos de Cooper, que nesta rede apenas representam a presença de autocitação. A Tabela 6 apresenta as informações sobre as referências mais citadas que estão representadas na rede de citações de artigos para referências (ver Figura 4, nós quadrados).

Como a análise de artigos mais citados não contempla trabalhos publicados mais recentemente, buscou-se verificar quais os trabalhos mais atuais destes autores. Considerando que há ocorrência de artigo mais citado até 2007, foram identificados todos os artigos publicados na base ISI, de todos os autores da relação dos 20 trabalhos mais citados, entre 2008 e 2010 (ver Anexo 1).

Tabela 6. Referências da rede de citações de artigos para referências.

\begin{tabular}{ll}
\multicolumn{1}{c}{ Referências } & \multicolumn{1}{c}{ Base de dados / editora } \\
\hline Cooper, Edgett e Kleinschmidt (1997) & ISI Web of Knowledge / Scopus / Industrial Research Institute \\
Cooper, Edgett e Kleinschmidt (1998a) & ISI Web of Knowledge / Scopus / Industrial Research Institute \\
Cooper, Edgett e Kleinschmidt (1998b) & Livro - Editora Perseus \\
Cooper, Edgett e Kleinschmidt (1999) & ISI Web of Knowledge / Scopus / Wiley Blackwell \\
Cooper, Edgett e Kleinschmidt (2000) & ISI Web of Knowledge / Scopus / Industrial Research Institute \\
Cooper, Edgett e Kleinschmidt (2001) & Livro - Editora Perseus \\
Griffin (1997) & ISI Web of Knowledge / Scopus / Wiley Blackwell \\
Henriksen e Traynor (1999) & ISI Web of Knowledge / Scopus / IEEExplore Digital Library \\
Linton, Walsh e Morabito (2002) & ISI Web of Knowledge / Scopus / Wiley Blackwell \\
Montoya-Weiss e Calantone (1994) & ISI Web of Knowledge / Scopus / Wiley Blackwell \\
Verhoef (2002) & ISI Web of Knowledge / Scopus / Elsevier \\
Wheelwright e Clark (1992) & Livro - Editora Free Press \\
\hline
\end{tabular}


A última rede desenhada foi a de colaboração entre os países, a qual mostra se os países produziram estes trabalhos acadêmicos com ou sem colaboração com outros países (ver Figura 5).

Alguns países produziram sem relacionar-se com outros países, como é o caso da Bélgica, Brasil, Finlândia, Israel, Japão, Malásia, Taiwan e África do Sul. Os Estados Unidos estiveram presentes em três redes menores, em parceria com países como Alemanha, Portugal, Nova Zelândia e Austrália. Os Estados Unidos apareceram também numa rede maior, composta por doze países, sendo praticamente quase todos da Europa.

No Brasil, publicados na base Scielo, foram encontrados artigos dos seguintes autores: Moraes e Laurindo (2003), Rabechini Junior, Maximiano e Martins (2005), Miguel (2008), Mayrink, MacedoSoares e Cavalieri (2009), Castro e Carvalho (2010a, b) e Padovani, Carvalho e Muscat (2010).

\section{Discussão}

Nessa seção, exploram-se os resultados da análise de conteúdo, que visa identificar os principais temas e lacunas na literatura. Esses resultados são então articulados com os da análise bibliométrica. A análise de conteúdo buscou evidenciar os principais processos e práticas elencados, bem como lacunas apontadas e tendências futuras.

$\mathrm{Na}$ análise dos trabalhos da amostra, observou-se que há uma lacuna de aplicação dos modelos e práticas da gestão de portfólio nas organizações (COOPER; EDGETT; KLEINSCHMIDT, 1997, 1999, 2001;
RABECHINI JUNIOR; MAXIMIANO; MARTINS, 2005; CASTRO; CARVALHO, 2010a, b). Aliada a essa constatação, os autores evidenciam uma série de dificuldades na utilização dos modelos e práticas, bem como nos resultados do processo de gestão de portfólio. Por exemplo, Fu-Chien (2002) e Ghasemzadeh, Archer e Iyogun (1999) argumentam que há dificuldade dos tomadores de decisão em lidar com a interpretação dos modelos mais complexos de gestão de portfólio, optando por utilizar apenas os aspectos financeiros, que têm maior domínio e conhecimento das análises. Por outro lado, Elonen e Artto (2003) argumentam que a tomada de decisão baseada nas relações de poder dos stakeholders tem impacto negativo no desempenho do portfólio.

Em decorrência das lacunas nos processos de gestão de portfólio nas organizações, os autores levantam uma série de problemas que resultam dessas lacunas tais como a síndrome de superalocação de recursos e a tomada de decisão baseada no poder dos stakeholders. Para Engwall e Jerbrant (2003), a síndrome de alocação de recursos é um dos problemas mais graves na gestão de multiprojetos. Esta questão tem sido apontada na literatura como um problema que surge antes mesmo da composição do portfólio. Cooper, Edgett e Kleinschmidt (1997) apresentaram um primeiro elenco de problemas críticos na gestão de portfólio no qual destacaram a falta de alinhamento estratégico, a seleção de portfólio de baixa qualidade, dificuldade de matar projetos, falta de foco, informações pouco acuradas para suportar o processo de decisão.

$\mathrm{Na}$ amostra pesquisada, considerando tanto os artigos da amostra inicial como as referências mais

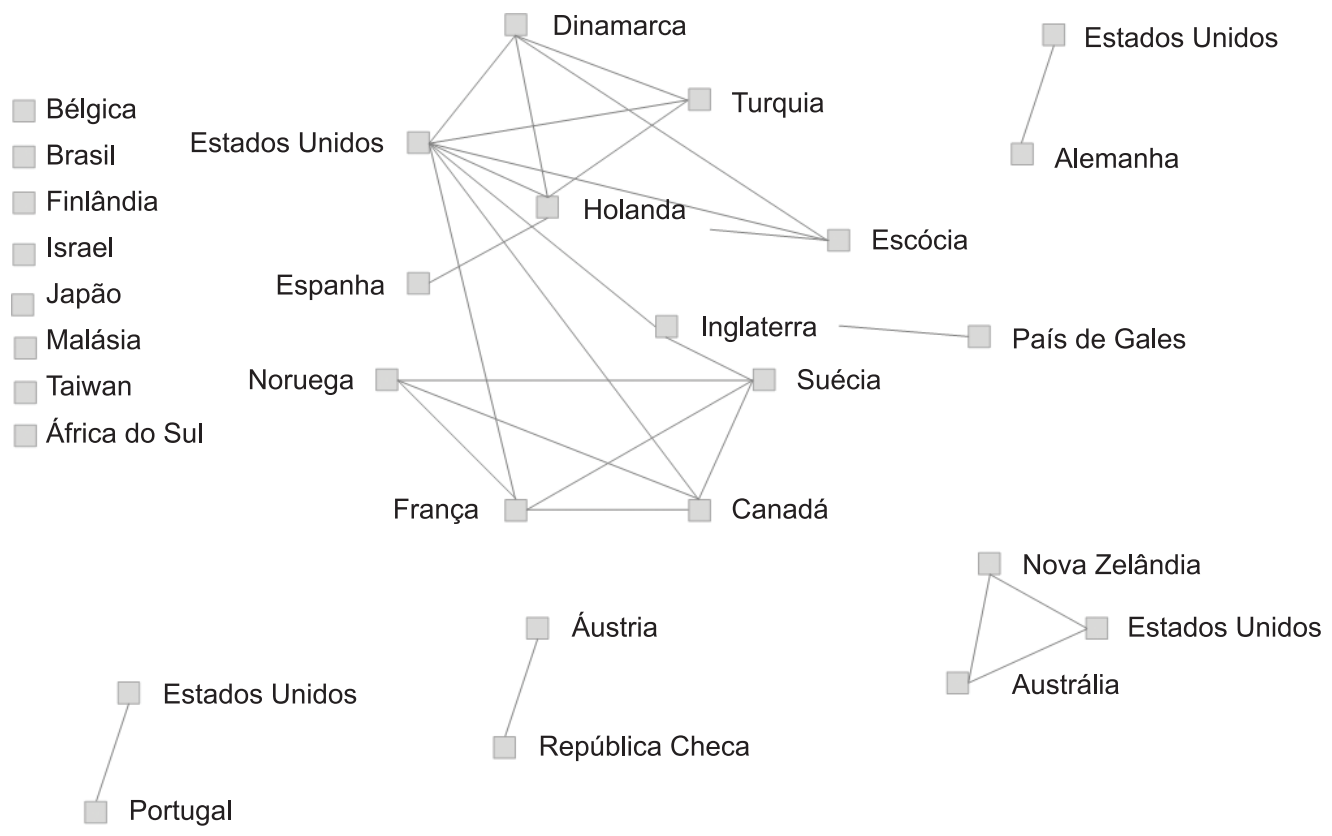

Figura 5. Rede de colaboração entre países 
citadas, foram identificados seis trabalhos com proposição de modelos ou que estabelecem processos (GHASEMZADEH; ARCHER; IYOGUN, 1999; COLDRICK et al., 2005; COOPER; EDGETT; KLEINSCHMIDT, 1998b; DETTBARN; IBSS; MURPHREE, 2005; PROJECT..., 2008; WHEELWRIGHT; CLARK, 1992), entre os quais, o único processo que é consenso é o de alocação de recursos. Na Figura 6, fizemos a conexão entre os processos conforme apresentados nos modelos.

É possível observar que o núcleo de processos que teve maior aderência entre os modelos (cinco ou seis menções) foram os seguintes: propostas de projetos, direcionadores estratégicos, identificação, avaliação, seleção, priorização, alocação de recursos, monitoramento \& controle. No grupo intermediário composto de dois processos, que receberam entre três e quatro menções, estão os processos de restrição de recursos e balanceamento. Há menor concentração (apenas uma ou duas menções) nos seguintes processos: metodologia para GPP, de categorização, ajuste e autorização.

Esses processos, suportados por um vasto elenco de ferramentas e técnicas encontrado na literatura estudada, foram classificados e sua síntese evolutiva está na Figura 7.

Foram também encontrados artigos que fazem uma vasta varredura das ferramentas, como o de Fu-Chien (2002). Na análise de conteúdo, adaptamos a classificação de ferramentas proposta em nove tipos. O primeiro tipo, denominado de métodos financeiros, engloba técnicas como valor presente líquido, a teoria de precificação de opções e opção real (real option), e é composto com o maior número de artigos relacionados (onze artigos). $\mathrm{O}$ segundo tipo de ferramentas, programação matemática, com nove artigos, é composto por ferramentas de otimização com restrições, tais como programação inteira, programação linear e não linear, programação por objetivo e programação dinâmica. O terceiro tipo são os modelos estatísticos tais como simulação de Monte Carlo, modelo probabilístico e rede bayesiana. Esses três grupos se distribuem ao longo de todo o período de análise, como ilustra a Figura 8. No início da série temporal, estão três tipos, o de ferramentas básicas (modelos de pontuação e listas de verificação), diagrama de bolhas e árvore de decisão. No período mais recente, observa-se a tendência de utilização de conceitos de lógica difusa (fuzzy), método de análise hierárquica (AHP), Análise Envoltória de Dados (DEA), ou mix destas duas técnicas (AHP e DEA) com abordagem difusa.

$\mathrm{Na}$ análise de conteúdo, buscou-se identificar os critérios utilizados para avaliação de portfólios. Identificamos vários trabalhos cuja temática envolvia os critérios de avaliação, quais sejam: Blau et al. (2004), Coldrick et al. (2005), Dickinson, Thornton e Graves (2001), Hart et al. (2003), Henriksen e Traynor (1999), Lin e Hsieh (2004), Linton, Walsh e Morabito (2002), Loch e Bode-Greuel (2001) e Trappey et al. (2009). Entre esses trabalhos, o que representou maior consenso foi o potencial de

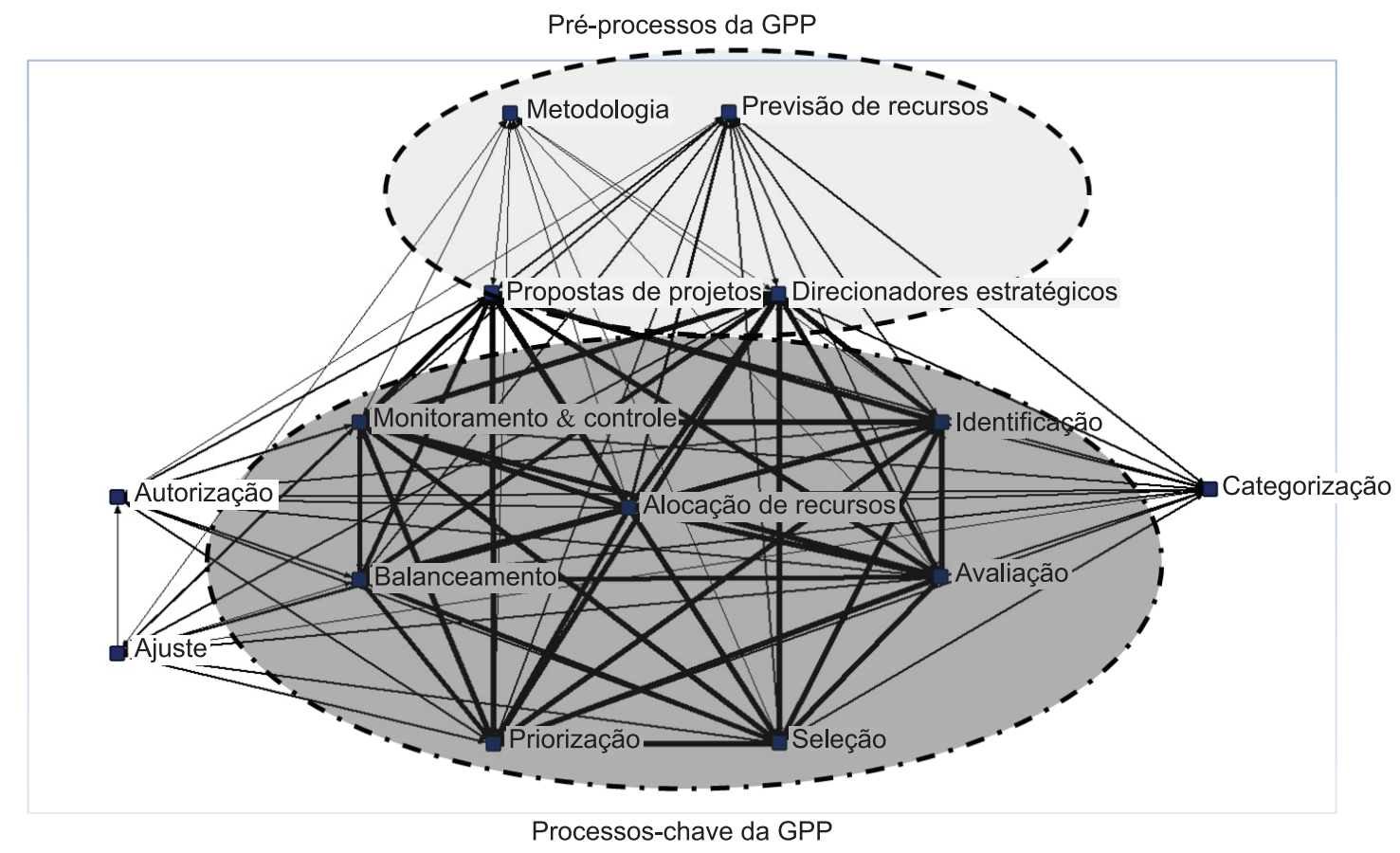

Figura 6. Conexão entre os processos de Gestão de Portfólio. Gráfico feito no NetDraw com base na análise de conteúdo. 
mercado, mencionado em oito dos nove trabalhos. Na sequência, aparecem dois critérios empatados: viabilidade econômico-financeira e riscos/ incertezas (cinco citações), seguido de viabilidade técnica (quatro citações). A Figura 8 mostra a evolução dos critérios no tempo, considerando-se o ano de publicação dos trabalhos que os mencionam.
A questão das interdependências entre os projetos do portfólio foi tratada separadamente, pois ora as interdependências aparecem como critério para a seleção ou ajuste do portfólio, ora aparecem nos modelos de otimização para alocação de recursos (COOPER; EDGETT; KLEINSCHMIDT, 1997a; DICKINSON; THORNTON; GRAVES, 2001;

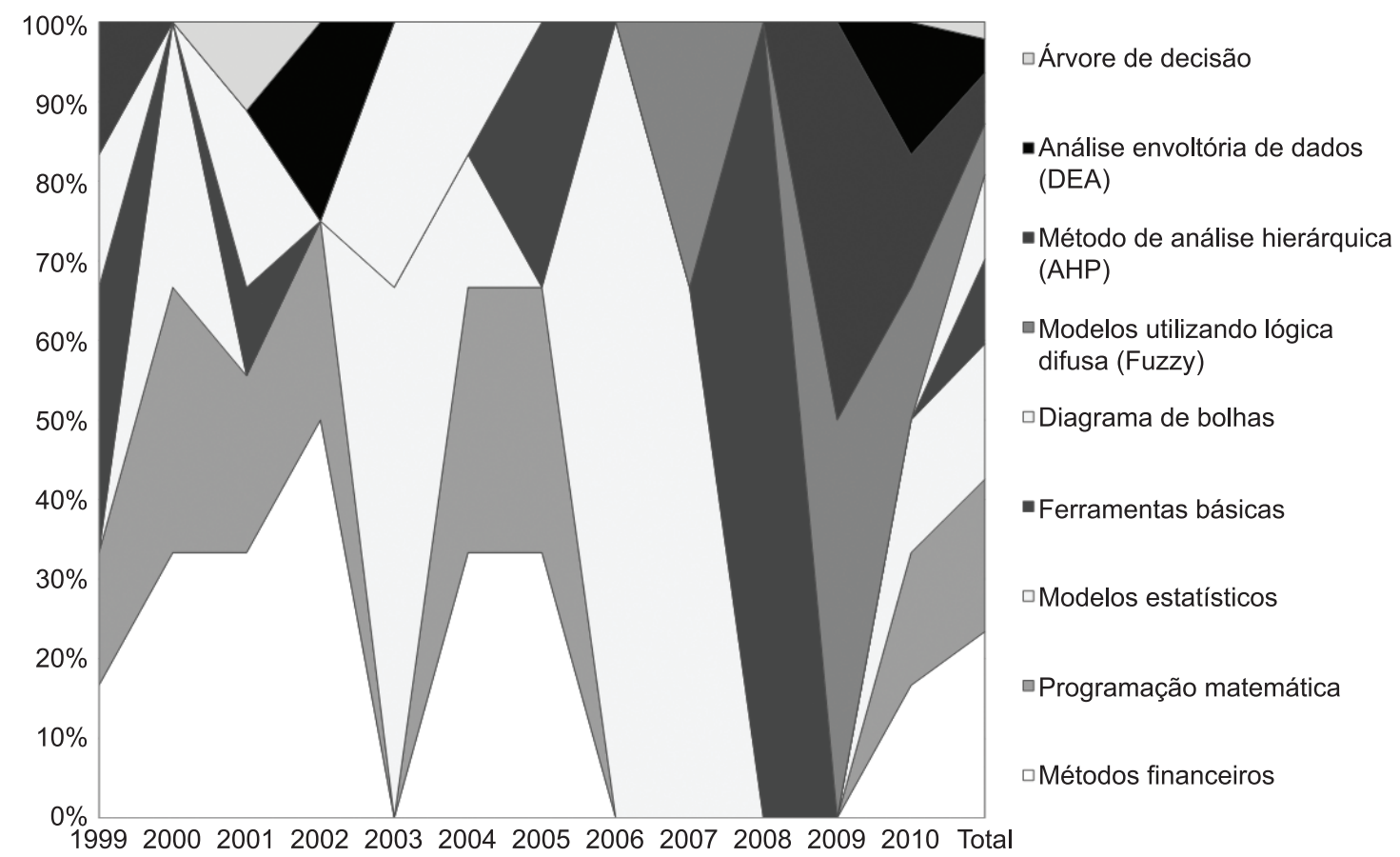

Figura 7. Evolução das ferramentas ao longo do tempo. Gráfico feito no Excel com base nas tabelas de análise de conteúdo.

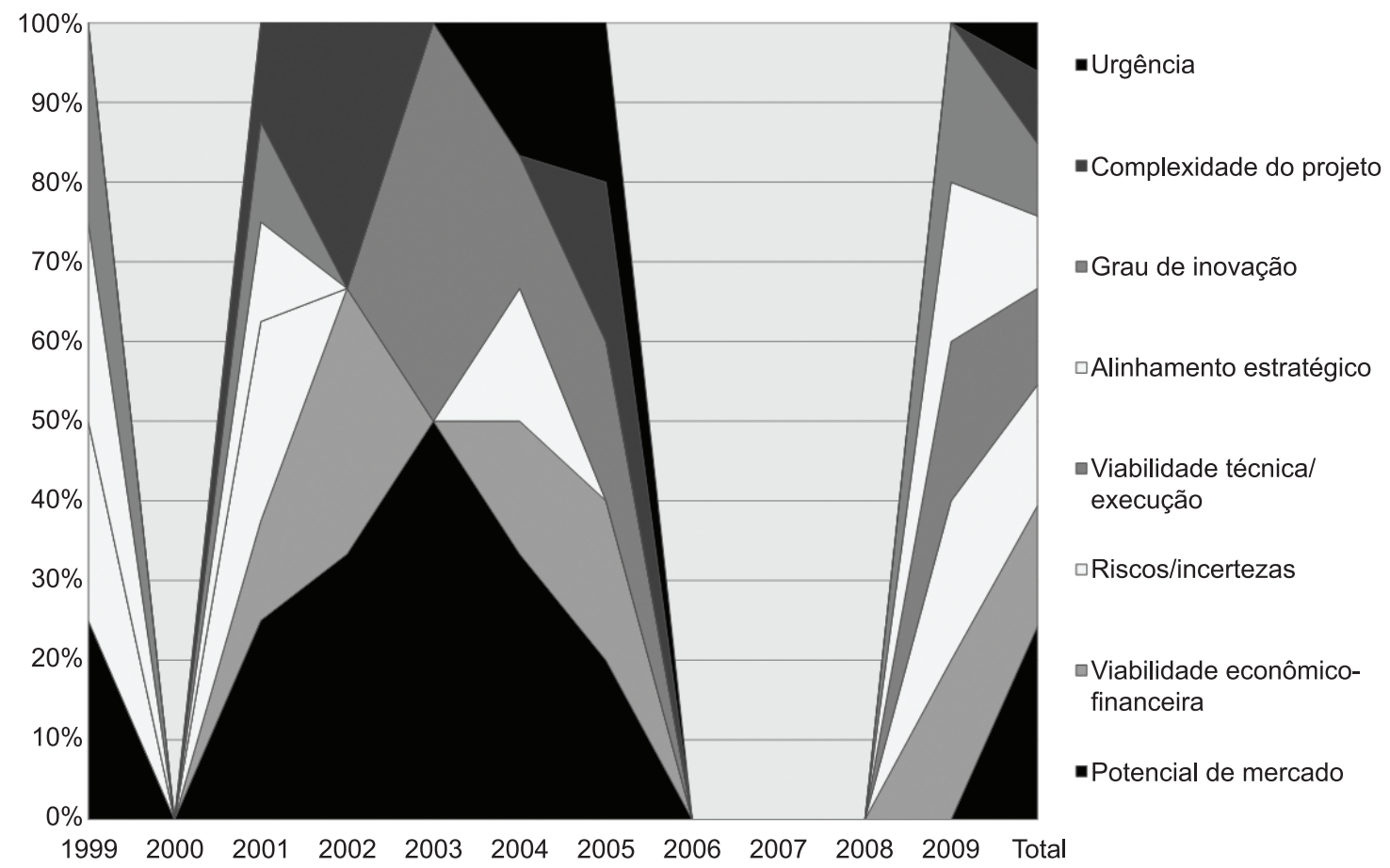

Figura 8. Evolução dos critérios de avaliação do portfólio. Gráfico feito no Excel com base nas tabelas de análise de conteúdo. 
BARDHAN; BAGCHI; SOUGSTAD, 2004, COLDRICK et al., 2005; GIROTRA; TERWIESCH; ULRICH, 2007; SANCHEZ; ROBERT; PELLERIN, 2008). Os tipos mais mencionados de interdependência na amostra estudada foram as interdependências técnicas, de recursos e temporais.

\section{Conclusões}

A abordagem híbrida de revisão de literatura, mesclando análise bibliométrica e de conteúdo, mostrou-se eficaz. Enquanto a bibliometria forneceu as bases para traçar um panorama da literatura, a análise de conteúdo permitiu a análise em profundidade dos estudos, extraindo os principais temas, lacunas e tendências. Apesar de os trabalhos sobre portfólio datarem da década de 1950, o tema gestão de portfólio de projetos é bem mais recente, e o volume mais significativo de citações data a partir de 1997. Essa tendência se alinha ao interesse crescente pela área de gerenciamento de projetos como um todo. Na base Scielo, encontramos artigos apenas a partir de 2003, o que mostra que se trata de um tema mais recente em periódicos nacionais.

O principal fórum de debate desta temática é composto pelos periódicos da área de inovação e desenvolvimento de produtos, dentre os quais se destacam Research Technology Management e Journal of Product Innovation Management. Dentre os periódicos nacionais, destacaram-se as Revistas Gestão \& Produção e Produção.

Observa-se uma concentração de trabalhos de Cooper e seus colaboradores, Cooper, Edgett e Kleinschmidt (1997, 1998a, 1999, 2002a, b, 2004a, b), com destaque para artigos relacionados ao framework stage gate ou ao levantamento (survey) sobre gestão de portfólio do grupo, que demonstra o alinhamento da teoria de gestão de portfólio de projetos com a de desenvolvimento de produtos.

Parte das citações foi alavancada por trabalhos como os de Montoya-Weiss e Calantone (1994) e de Griffin (1997), ambos listados entre os artigos clássicos do periódico Journal of Product Innovation Management no artigo de Biemans, Griffin e Moenart (2010) e relacionados na rede de artigos para referência. Observou-se uma convergência dos modelos de gestão de portfólio no que concerne aos seguintes processos-chave: propostas de projetos, direcionadores estratégicos; identificação; avaliação; seleção; priorização; alocação de recursos; e monitoramento \& controle. A análise das ferramentas demonstrou que, enquanto algumas categorias (métodos financeiros, programação matemática e modelos estatísticos) são encontradas em todo o período de análise, técnicas mesclando abordagens tradicionais e conceitos de modelagem difusa (fuzzy) aparecem como uma tendência mais recente. Quanto aos critérios para seleção dos portfólios, há uma predominância de três critérios na amostra estudada: potencial de mercado, viabilidade econômico-financeira e riscos/ incertezas.

Vale destacar as limitações impostas pelas opções metodológicas desta pesquisa. A primeira refere-se à amostra inicial extraída da base de dados internacional (ISI Web of Science) e da nacional (Scielo) e por analisar apenas artigos e periódicos. No entanto, a amostra inicial foi expandida por meio da rede de artigos para referências, que envolveram livros e outros tipos de publicações de outras bases de dados, tais como Wiley Blackwell, Industrial Research Institute, IEEExplore Digital Library e Elsevier.

Outra limitação refere-se ao fato de que, na análise de citações, os artigos mais recentes têm desvantagem em detrimento dos mais antigos, criando um viés temporal.

Como sugestão de temas futuros, aponta-se a lacuna de trabalhos que busquem evidenciar o impacto da gestão de portfólio no desempenho dos projetos e organizacionais a partir de abordagens quantitativas e confirmatórias, dado que há estudos de caso que exploram esse tipo de relação. Adicionalmente, no contexto brasileiro, há carência de estudos que explorem o estado da prática; encontrou-se apenas o levantamento de Castro e Carvalho (2010a), mas a amostra é limitada a 31 empresas.

\section{Agradecimentos}

Os autores gostariam de agradecer as sugestões recebidas dos referees anônimos da revista e aos órgãos de fomento CAPES e CNPq o apoio recebido.

\section{Referências}

ABHARY, S. et al. Common Sequence Variation in the VEGFA Gene Predicts Risk of Diabetic Retinopathy. Investigative Ophthalmology \& Visual Science, v. 50, n. 12, p. 5552-5558, 2009. http://dx.doi.org/10.1167/ iovs.09-3694

ANDERSSON, B. et al. The prevalence of and perceived risks from drug use among teenagers in 33 European countries. Journal of Substance Use, v. 14, n. 3-4, p. 189-196, 2009. http://dx.doi.org/10.1080/14659890802668805

BARDHAN, I.; BAGCHI, S.; SOUGSTAD, R. Priorizing a portfolio of information technology investment projects. Journal of Management Information Systems, v.21, n. 2, p. 33-60, 2004.

BAGCHI, S.; ZOLTOWSKI, C. B.; OAKES, W. C. Work in Progress - Impact of Research Technologies on Service Learning. IEEE Frontiers in Education Conference, v. 1-3, p. 1612-1613, 2008.

BIEMANS, W.; GRIFFIN, A.; MOENART, R. In search of the classics: A study of the impact of JPIM papers from 1984 to 2003. Journal of Product Innovation Management, v. 27, n. 4, p. 461-484, 2010. http:// dx.doi.org/10.1111/j.1540-5885.2010.00730.x

BLAU, G. E. et al. Managing a portfolio of interdependent new product candidates in the pharmaceutical industry. Journal of Product Innovation Management, 
v. 21, n. 4, p. 227-245, 2004. http://dx.doi. org/10.1111/j.0737-6782.2004.00075.x

BORGATTI, S.; EVERETT, M.; FREEMAN, L. Ucinet for Windows: software for social network analysis. Analytic Technologies, 2002.

BRUMMER, V.; KONNOLA, T.; SALO, A. Foresight within ERA-NETs: Experiences from the preparation of an international research program. Technological Forecasting and Social Change, v. 75, n. 4, p. 483-495, 2008. http://dx.doi.org/10.1016/j. techfore.2008.02.005

CARVALHO, M. M.; RABECHINI JUNIOR, R. Construindo competências para gerenciar projetos: teoria e casos. São Paulo: Atlas, 2008.

CASTRO, H. G.; CARVALHO, M. M. Gerenciamento do portfolio de projetos: um estudo exploratório. Gestão \& Produção, v.17, n. 2, p. 283-296, 2010a. http://dx.doi. org/10.1590/S0104-530X2010000200006

CASTRO, H. G.; CARVALHO, M. M. Gerenciamento do portfólio de projetos (PPM): estudos de caso. Produção, v. 20, n. 3, p. 303-321, 2010b. http://dx.doi.org/10.1590/ S0103-65132010005000044

COLDRICK, S. et al. An R\&D options selection model for investment decisions. Technovation, v. 25, n. 3, p. 185-193, 2005. http://dx.doi.org/10.1016/ S0166-4972(03)00099-3

COOPER, R. G. Perspective: The Stage-Gate (R) idea-to-launch process-update, what's new, and NexGen systems. Journal of Product Innovation Management, v. 25, n. 3, p. 213-232, 2008. http:// dx.doi.org/10.1111/j.1540-5885.2008.00296.x

COOPER, R. G. How companies are reinventing their idea-to-launch methodologies. Research Technology Management, v. 52, n. 2, p. 47-57, 2009.

COOPER, R. G.; EDGETT, S. J. Overcoming the crunch in resources for new product development. Research Technology Management, v. 46, n. 3, p. 48-59, 2003. COOPER, R. G.; EDGETT, S. J. Overcoming the crunch in resources for new product development. Research Technology Management, v. 51, n. 2, p. 47-58, 2008. COOPER, R. G.; EDGETT, S. J.; KLEINSCHMIDT, E. J. Maximizing productivity in product innovation. Research Technology Management, v. 40, n. 5, p. 16-29, 1997. COOPER, R. G.; EDGETT, S. J.; KLEINSCHMIDT, E. J. Best practices for managing R\&D portfolios. Research Technology Management, v. 41, n. 4, p. 20-34, 1998a. COOPER, R. G.; EDGETT, S. J.; KLEINSCHMIDT, E. J. Portfolio Management for new Product. M.A. Perseus, 1998b.

COOPER, R. G.; EDGETT, S. J.; KLEINSCHMIDT, E. J. New product portfolio management: practices and performance. Journal of Product Innovation Management, v. 16, n. 4, p. 333-350, 1999. http:// dx.doi.org/10.1016/S0737-6782(99)00005-3

COOPER, R. G.; EDGETT, S. J.; KLEINSCHMIDT, E. J. New problems, new solutions: Making portfolio management more effective. Research Technology Management, v. 43, n. 2, p. 18-34, 2000.

COOPER, R. G.; EDGETT, S. J.; KLEINSCHMIDT, E. J. Portfolio management for new products. M.A. Perseus, 2001.
COOPER, R. G.; EDGETT, S. J.; KLEINSCHMIDT, E. J. Optimizing the stage-gate process: What best-practice companies do-I. Research Technology Management, v. 45 , n. 5 , p. 21-28, 2002a.

COOPER, R. G.; EDGETT, S. J.; KLEINSCHMIDT, E. J. Optimizing the stage-gate process: What best-practice companies do-II? Research Technology Management, v. 45, n. 5 , p. 21-28, 2002 b.

COOPER, R. G.; EDGETT, S. J.; KLEINSCHMIDT, E. J. Benchmarking best npd practices-I. Research Technology Management, v. 47, n. 1, p. 31, 2004 a.

COOPER, R. G.; EDGETT, S. J.; KLEINSCHMIDT, E. J. Benchmarking best npd practices-II. Research Technology Management, v. 47, n. 1, p. 31, 2004 b.

DESAI, V. et al. Enhancement of primary education using EDUSAT: Rajiv Gandhi project for EDUSAT supported elementary education network (RGPEEE) overview. ACTA Astronautica, v. 65, n. 9-10, p. 1440-1445, 2009. http://dx.doi.org/10.1016/j.actaastro.2009.03.086

DETTBARN, J. L.; IBBS, W. P. E.; MURPHREE, L. P. E. Capital project portfolio management for federal real property. Journal of Management in Engineering, v. 21, n. 1, p. 44-53, 2005. http://dx.doi.org/10.1061/ (ASCE)0742-597X(2005)21:1(44)

DICKINSON, M. W.; THORNTON, A. C.; GRAVES, S. Technology portfolio management: Optimizing interdependent projects over multiple time periods. IEEE Transactions on Engineering Management, v. 48, n. 4 , p. 518-527, 2001. http://dx.doi.org/10.1109/17.969428

DUSO, T.; PENNINGS, E.; SELDESLACHTS, J. Learning dynamics in research alliances: A panel data analysis. Research Policy, v. 39, n. 6, p. 776-789, 2010. http:// dx.doi.org/10.1016/j.respol.2010.03.002

ELEKES, Z. et al. Changes in perceived risk of different substance use by ranking order of drug attitudes in different ESPAD-countries. Journal of Substance Use, v. 14, n. 3-4, p. 197-210, 2009. http://dx.doi. org/10.1080/14659890802668813

ELONEN, S.; ARTTO, K. A. Problems in managing internal development projects in multi-project environments. International Journal of Project Management, v. 21, n. 6, p. 395-402, 2003. http://dx.doi.org/10.1016/ S0263-7863(02)00097-2

ENGLUND, M. et al. Multivariate latent risk: A credibility approach. Astin Bulletin, v. 38, n. 1, p. 137-146, 2008. http://dx.doi.org/10.2143/AST.38.1.2030406

ENGWALL, M.; JERBRANT, A. The resource allocation syndrome: The prime challenge of multi-project management. International Journal of Product Management, v. 21, n. 6, p. 403-409, 2003.

FAUCETT, W. A. et al. A model program to increase translation of rare disease genetic tests: collaboration, education, and test translation program. Genetics in Medicine, v. 10, n. 5, p. 343-348, 2008. http://dx.doi. org/10.1097/GIM.0b013e318172837c

FOSTER, D. P.; HART, S. An Operational Measure of Riskiness. Journal of Political Economy, v. 117, n. 5, p. 785-814, 2009. http://dx.doi.org/10.1086/644840

FU-CHIEN, C. A portfolio evaluation framework for selection $R \& D$ projects. R\&D Management, 
v. 32, n. 4, p. 359-368, 2002. http://dx.doi. org/10.1111/1467-9310.00266

GIROTRA, K.; TERWIESCH, C.; ULRICH, K. T. Valuing $R \& D$ projects in a portfolio: evidence from the pharmaceutical industry. Management Science, v. 53, p. 1452-1466, 2007. http://dx.doi.org/10.1287/ mnsc. 1070.0703

GRIFFIN, A. PDMA Research on new product development practices: updating trends and benchmarking best practices. Journal of Product Innovation Management, v. 14, n. 6, p. 429-458, 1997. http://dx.doi.org/10.1016/ S0737-6782(97)00061-1

GUPTA, A. et al. Use of collaborative technologies and knowledge sharing in co-located and distributed teams: Towards the 24-h knowledge factory. Journal of Strategic Information Systems, v. 18, n. 3, p. 147-161, 2009. http://dx.doi.org/10.1016/j.jsis.2009.07.001

GUPTA, A. et al. Haplotype Inferring via Galled-Tree Networks Is NP-Complete. Journal of Computational Biology, v. 17, n. 10, p. 1435-1449, 2010. http://dx.doi. org/10.1089/cmb.2009.0117

GUPTA, A. et al. Federated Access to Heterogeneous Information Resources in the Neuroscience Information Framework (NIF). Neuroinformatics, v. 6, n. 3, p. 205-217, 2008. http://dx.doi.org/10.1007/ s12021-008-9033-y

GUSTAFSSON, J.; SALO, A. Contingent portfolio programming for the management of risky projects. Operations Research, v. 53, n. 6 p. 946-956, 2005. http://dx.doi.org/10.1287/opre.1050.0225

GUSTAFSSON, J. et al. ALL-TIMES - A European Project on Integrating Timing Technology. Communications in Computer and Information Science, v. 17, p. 445-459, 2008. http://dx.doi. org/10.1007/978-3-540-88479-8_31

GHASEMZADEH, F.; ARCHER, N., IYOGUN, P. A zero-one model for project portfolio selection and scheduling. Journal of the operational Research Society, v. 50, n. 7, p. 745-755, 1999. http://dx.doi. org/ 10.1057/palgrave.jors.2600767

HART, S. et al. Industrial companies 'evaluation criteria in new product development gates. Journal of Product Innovation Management, v. 20, n. 1, p. 20:22-36, 2003.

HENRIKSEN, A. D.; TRAYNOR, A. J. A practical R\&D project-selection scoring tool. IEEE Transaction on Engineering Management, v. 46, n. 2, p. 158-170, 1999. http://dx.doi.org/10.1109/17.759144

HOGAN, A. et al. The Health Impact of a Hearing Disability on Older People in Australia. Journal of Aging and Health, v. 21, n. 8, p. 1098-1111, 2009. http://dx.doi. org/10.1177/0898264309347821

IKPAAHINDI, L. An Overview of Bibliometrics: its Measurements, Laws and their Applications. Libri, v. 35, n. 2, p.163-177, 1985.

JARIMO, T.; SALO, A. Multicriteria Partner Selection in Virtual Organizations with Transportation Costs and Other Network Interdependencies. IEEE Transactions on Systems Man and Cybernetics, v. 39, n. 1, p. 124-129, 2009. http://dx.doi.org/10.1109/ TSMCC.2008.2007251
KAUFFMAN, R. J.; SOUGSTAD, R. Risk Management of Contract Portfolios in IT Services: The Profit-at-Risk Approach. Journal of Management Information Systems, v. 25, n. 1, p. 17-48, 2008. http://dx.doi. org/10.2753/MIS0742-1222250102

KER, H. W.; LEE, Y. H.; LIN, C. H. Prediction Models for Transverse Cracking of Jointed Concrete Pavements Development with Long-Term Pavement Performance Database. Transportation Research Record, n. 2068, p. 20-31, 2008. http://dx.doi.org/10.3141/2068-03

KESSLER, M. M. Bibliographic coupling between scientific papers. American Documentation, v. 14, n. 10-25, 1963.

KESTER, L.; HULTINK, E. J.; LAUCHE, K. Portfolio decision-making genres: A case study. Journal of Engineering and Technology Management, v. 26, n. 4, p. 327-341, 2009. http://dx.doi.org/10.1016/j. jengtecman.2009.10.006

KETTUNEN, J.; SALO, A.; BUNN, D. W. Optimization of Electricity Retailer's Contract Portfolio Subject to Risk Preferences. IEEE Transactions on Power Systems, v. 25 , n. 1, p. 117-128, 2010. http://dx.doi.org/10.1109/ TPWRS.2009.2032233

LANGERAK, F.; HULTINK, E. J. The effect of new product development acceleration approaches on development speed: A case study. Journal of Engineering and Technology Management, v. 25, n. 3, p. 157-167, 2008. http://dx.doi.org/10.1016/j.jengtecman.2008.06.004

LERCH, R. N. et al. Overview of the Mark Twain Lake/Salt River Basin Conservation Effects Assessment Project. Journal of Soil and Water Conservation, v. 63, n. 6, p. 345-359, 2008. http://dx.doi.org/10.2489/jswc.63.6.345

LI, X. et al. Real-time storm detection and weather forecast activation through data mining and events processing. Arth Science Informatics, v. 1, n. 2, p. 49-57, 2008. http://dx.doi.org/10.1007/s12145-008-0010-7

LIESIO, J.; MILD, P.; SALO, A. Robust portfolio modeling with incomplete cost information and project interdependencies. European Journal of Operational Research, v. 190, n. 3, p. 679-695, 2008. http://dx.doi. org/10.1016/j.ejor.2007.06.049

LIN, C. H. A model using home appliance ownership data to evaluate recycling policy performance. Resources Conservation and Recycling, v. 52, n. 11, p. 1322-1328, 2008. http://dx.doi.org/10.1016/j. resconrec.2008.07.015

LIN, C. H.; HSIED, R. J. A fuzzy decision support for strategic portfolio management. Decision Support Systems, v. 38, n. 3, p. 383-398, 2004. http://dx.doi. org/10.1016/S0167-9236(03)00118-0

LIN, C. H.; HSU, M. L. Holistic decision system for human resource capability identification. Industrial Management \& Data Systems, v. 110, n. 1-2, p. 130-248, 2010.

LIN, C. H.; HUANG, T. H.; SHAW, D. Applying Water Quality Modeling to Regulating Land Development in a Watershed. Water Resources Management, v. 24, n. 4, p. 629-640, 2010. http://dx.doi.org/10.1007/ s11269-009-9462-x

LIN, C. H.; WEN, L.; TSAI, Y. M. Applying decision-making tools to national e-waste recycling policy: An example of Analytic Hierarchy Process. Waste Management, 
v. 30, n. 5, p. 863-869, 2010. http://dx.doi.org/10.1016/j. wasman.2009.11.012

LIN, C. H. et al. Design for Usability on Supply Chain Management Systems Implementation. Human Factors and Ergonomics in Manufacturing, v. 19, n. 5, p. 378-403, 2009. http://dx.doi.org/10.1002/hfm.20171

LIN, C. H. et al. Pursuing excellence in firm core knowledge through intelligent group decision support system. Industrial Management \& Data Systems, v. 108, n. 3-4, p. 277-296, 2008. http://dx.doi. org/10.1108/02635570810858723

LINDSTEDT, M.; LIESIO, J.; SALO, A. Participatory development of a strategic product portfolio in a telecommunication company. International Journal of Technology Management, v. 42, n. 3, p. 250-266, 2008. http://dx.doi.org/10.1504/IJTM.2008.018106

LINT, O.; PENNINGS, E. An option approach to the new product development process: a case study at Philips Electronics. R\&D Management, v. 31, n. 2, p. 163-172, 2001. http://dx.doi. org/10.1111/1467-9310.00206

LINTON, J. D.; WALSH, S. T.; MORABITO, J. Analysis, ranking and selection of $\mathrm{R} \& \mathrm{D}$ projects in a portfolio. R\&D Management, v. 32, n. 2, p. 139-148, 2002. http://dx.doi.org/10.1111/1467-9310.00246

LOCH, C. H.; BODE-GREUEL, K. Evaluating growth options as sources of value for pharmaceutical research projects. R\&D Management, v. 31, n. 2, p. 231-248, 2001. http:// dx.doi.org/10.1111/1467-9310.00212

MAYRINK, E. F.; MACEDO-SOARES, T. D. L. A.; CAVALIERI, A. Adequação estratégica de projetos: o caso da Eletronuclear. Revista de Administração Pública, v. 43, n. 6, p. 1217-1250, 2009. http://dx.doi. org/10.1590/S0034-76122009000600002

MEKSENAS, P. Aspectos metodológicos da pesquisa empírica. Periódico Espaço acadêmico, n. 78, 2009.

MIGUEL, P. C. Implementação da gestão de portfolio de novos produtos: um estudo de caso. Produção, v. 18, n. 2, p. 388-404, 2008. http://dx.doi.org/10.1590/ S0103-65132008000200014

MIKKOLA, J. H. Portfolio management of R\&D projects: implications for innovation management. Technovation, v. 21, n. 7, p. 423-435, 2001. http://dx.doi.org/10.1016/ S0166-4972(00)00062-6

MILD, P.; SALO, A. Combining a Multiattribute Value Function with an Optimization Model: An Application to Dynamic Resource Allocation for Infrastructure Maintenance. Decision Analysis, v. 6, n. 3, p. 139-152, 2009. http://dx.doi.org/10.1287/ deca.1090.0143

MILLER, P. Role of pharmacoeconomic analysis in R\&D decision making - when, where, how? Pharmacoeconomics, v. 23, n. 1, p. 1-12, 2005. http:// dx.doi.org/10.2165/00019053-200523010-00001

MILLER, P.; CHOMCYNOVA, P.; BECK, F. Predicting Teenage Beliefs Concerning the Harm Alcohol and Cannabis Use may do in Eight European Countries. Journal of Substance Use, v. 14, n. 6, p. 364-374, 2009. http://dx.doi.org/10.3109/14659890802668789

MOJSILOVIĆ, A. et al. A logistic regression framework for information technology outsourcing lifecycle management. Computers \& Operations Research, v. 34, n. 12 , p. 3609-3627, 2007. http://dx.doi.org/10.1016/j. cor.2006.01.018

MONTOYA-WEISS, M. M.; CALANTONE, W. R. Determinants of new product performance: a review and meta-analysis. Journal of Product Innovation Management, v. 11, n. 5, p. 397-417, 1994. http:// dx.doi.org/10.1016/0737-6782(94)90029-9

MORAES, R. O.; LAURINDO, F. J. B. Um estudo de caso de gestão de portfolio de projetos de tecnologia da informação. Gestão \& Produção, v.10, n.3, p. 311-328, 2003. http://dx.doi.org/10.1590/ S0104-530X2003000300007

NEELY, A. The evolution of performance measurement research: developments in the last decade and a research agenda for the next. International Journal of Operations \& Production Management, v. 25 , n. 12 , p. 1264-1277, 2005. http://dx.doi. org/10.1108/01443570510633648

PADOVANI, M.; CARVALHO, M. M.; MUSCAT, A. R. N. Seleção e alocação de recursos em portfólio de projetos: estudo de caso no setor químico. Gestão \& Produção, v. 17, n. 1, p. 157-180, 2010. http://dx.doi. org/10.1590/S0104-530X2010000100013

PENG, P. C. et al. Testing of Holes in Concrete Using Gamma Radiography. Materials Evaluation, v. 67, n. 3, p. 327-332, 2009

PRASAD, S.; TATA, J. Publication patterns concerning the role of teams/groups in the information systems literature from 1990 to 1999. Information \& Management, v. 42, n. 8, p. 1137-1148, 2005. http://dx.doi.org/10.1016/j. im.2005.01.003

PROJECT MANAGEMENT INSTITUTE - PMI. The standard for portfolio management. PMI, 2008.

RABECHINI JUNIOR, R.; MAXIMIANO, A. C. A.; MARTINS, V. A. A adoção de gerenciamento de portfólio como uma alternativa gerencial: o caso de uma empresa prestadora de serviço de interconexão eletrônica. Produção, v. 15, n.3, p. 416-433, 2005.

RAMOS-RODRIGUEZ, A. R., RUIZ-NAVARRO, J. Changes in the intellectual structure of strategic management research: A bibliometric study of the strategic management journal, 1980-2000. Strategic Management Journal, v. 25, n. 10, p. 981-1004, 2004. http://dx.doi.org/10.1002/smj.397

ROGERS, M. J.; GUPTA, A.; MARANAS, C. D. Real options based analysis of optimal pharmaceutical research and development portfolios. Industrial \& Engineering Chemistry Research, v. 41, n. 25, p. 6607-6620, 2002. http://dx.doi.org/10.1021/ie020385p

ROZENFELD, H. et al. Gestão de desenvolvimento de produto: uma referência para a melhoria do processo. São Paulo: Saraiva, 2006. p. 1-101.

SANCHEZ, H.; ROBERT, B.; PELLERIN, R. A project portfolio risk-opportunity identification framework. Project Management Journal, v. 39, n. 32, p. 97-109, 2008. http://dx.doi.org/10.1002/pmj.20072 SARGAONKAR, A. P.; GUPTA, A.; DEVOTTA, S. Evaluation of Monitoring Sites for Protection of Groundwater in an Urban Area. Water Environment 
Research, v. 80, n. 11, p. 2157-2164, 2008. http:// dx.doi.org/10.2175/106143008X304695

SCHILDT, H. A. Sitkis: Software for Bibliometric Data Management and Analysis. Helsinki: Institute of Strategy and International Business, 2002. v. 6.1.

SIDDIQI, S. N. Project valuation and power portfolio management in a competitive market. IEEE Transactions on Power Systems, v. 15, n. 1, p. 116-121, 2000. http:// dx.doi.org/10.1109/59.852109

SINGH, M. K.; EINSTADTER, D.; LAWRENCE, R. A structured women's preventive health clinic for residents: a quality improvement project designed to meet training needs and improve cervical cancer screening rates. Quality \& Safety in Health Car, v. 19, n. 5, p. 45, 2010. http://dx.doi.org/10.1136/qshc.2009.033274

TRAPPEY, C. V. et al. A strategic product portfolio management methodology considering $R \& D$ resource constraints for engineering-to-order industries. International Journal of Technology Management, v. 48, n. 2, p. 258-276, 2009. http://dx.doi.org/10.1504/ IJTM.2009.024919

VARMA, V. A. et al. A framework for addressing stochastic and combinatorial aspects of scheduling and resource allocation in pharmaceutical R\&D pipelines. Computers \& Chemical Engineering, v. 32, n. 4-5, p. 1000-1015, 2008. http://dx.doi.org/10.1016/j. compchemeng.2007.05.006

VAN BEKKUM, S.; PENNINGS, E.; SMITH, H. A real options perspective on $\mathrm{R} \& \mathrm{D}$ portfolio diversification.
Computers \& Chemical Engineering, v. 38, n. 7, p. 1150-1158, 2009.

VERHOEF, C. Quantitative IT portfolio management. Science of computer programming, v. 45, n. 1, p. 1-96, 2002. http://dx.doi.org/10.1016/ S0167-6423(02)00106-5

VIJAY, R.; SARGAONKAR, A.; GUPTA, A. A hydrodynamic approach to address Yamuna riverbed development in Delhi. Canadian Journal of Civil Engineering, v. 36, n. 7, p. 1155-1163, 2009. http:// dx.doi.org/10.1139/L09-059

WANG, J. T.; HWANG, W. I. A fuzzy set approach for portfolio selection using a real options valuations model. Omega-International Journal of Management Science, v. 35, n. 3, p. 247-257, 2007. http://dx.doi. org/10.1016/j.omega.2005.06.002

WEHRMANN, A.; ZIMMERMANN, S. Integrated ex-ante risk-return evaluation of IT investments. Wirschaftsinformatik, v. 47, n. 4, p. 247-257, 2005. http://dx.doi.org/10.1007/BF03254912

WHEELWRIGHT, S. C.; CLARK, K. B. Revolutionizing product development: quantum leaps in speed efficiency and quality. New York: Free Press, 1992.

WHITE, H.; McCAIN, K. Visualizing a discipline: An author co-citation analyzes of information science, 1972-1995. Journal of the American Society for Information Science, v. 49, n. 4, p. 327-355, 1998. 
Anexo 1. Lista detalhada de artigos.

\begin{tabular}{|c|c|c|}
\hline Artigo & $\begin{array}{r}\text { Título } \\
\end{array}$ & Periódico \\
\hline Abhary et al. (2009) & $\begin{array}{l}\text { Common Sequence Variation in the VEGFA Gene } \\
\text { Predicts Risk of Diabetic Retinopathy }\end{array}$ & $\begin{array}{c}\text { Investigative Ophthalmology \& } \\
\text { Visual Science }\end{array}$ \\
\hline Andersson et al. (2009) & $\begin{array}{l}\text { The prevalences of and perceived risks from drug } \\
\text { use among teenagers in } 33 \text { European countries }\end{array}$ & Journal of Substance Use \\
\hline $\begin{array}{l}\text { Bagchi, Zoltowski e } \\
\text { Oakes (2008) }\end{array}$ & $\begin{array}{l}\text { Work In Progress - Impact of Research } \\
\text { Technologies on Service Learning }\end{array}$ & $\begin{array}{l}\text { IEEE Frontiers in Education } \\
\text { Conference }\end{array}$ \\
\hline $\begin{array}{l}\text { Brummer, Konnola e } \\
\text { Salo (2008) }\end{array}$ & $\begin{array}{l}\text { Foresight within ERA-NETs: Experiences from the } \\
\text { preparation of an international research program }\end{array}$ & $\begin{array}{l}\text { Technological Forecasting and } \\
\text { Social change }\end{array}$ \\
\hline Cooper e Edgett (2008) & Maximizing productivity in product innovation & Research-Technology Management \\
\hline Cooper (2008) & $\begin{array}{l}\text { Perspective: The Stage-Gate (R) idea-to-launch } \\
\text { process-update, what's new, and NexGen systems }\end{array}$ & $\begin{array}{c}\text { Journal of Product Innovation } \\
\text { Management }\end{array}$ \\
\hline Cooper (2009) & $\begin{array}{l}\text { How companies are reinventing their idea-to-launch } \\
\text { methodologies }\end{array}$ & Research-Technology Management \\
\hline Desai et al. (2009) & $\begin{array}{l}\text { Enhancement of primary education using EDUSAT: } \\
\text { Rajiv Gandhi project for EDUSAT supported } \\
\text { elementary education network (RGPEEE) overview }\end{array}$ & ACTA Astronautica \\
\hline $\begin{array}{c}\text { Duso, Pennings e } \\
\text { Seldeslachts (2010) }\end{array}$ & $\begin{array}{l}\text { Learning dynamics in research alliances: A panel } \\
\text { data analysis }\end{array}$ & Research Policy \\
\hline Elekes et al. (2009) & $\begin{array}{l}\text { Changes in perceived risk of different substance } \\
\text { use by ranking order of drug attitudes in different } \\
\text { ESPAD-countries }\end{array}$ & Journal of Substance Use \\
\hline Englund et al. (2008) & Multivariate latent risk: A credibility approach & Astin Bulletin \\
\hline Faucett et al. (2008) & $\begin{array}{l}\text { A model program to increase translation of rare } \\
\text { disease genetic tests: collaboration, education, and } \\
\text { test translation program }\end{array}$ & Genetics in Medicine \\
\hline Foster e Hart (2009) & An Operational Measure of Riskiness & Journal of Political Economy \\
\hline Gupta et al. (2009) & $\begin{array}{l}\text { Use of collaborative technologies and knowledge } \\
\text { sharing in co-located and distributed teams: } \\
\text { Towards the 24-h knowledge factory }\end{array}$ & $\begin{array}{c}\text { Journal of Strategic Information } \\
\text { Systems }\end{array}$ \\
\hline Gupta et al. (2008) & $\begin{array}{l}\text { Federated Access to Heterogeneous Information } \\
\text { Resources in the Neuroscience Information } \\
\text { Framework (NIF) }\end{array}$ & Neuroinformatics \\
\hline Gupta et al. (2010) & $\begin{array}{l}\text { Haplotype Inferring via Galled-Tree Networks Is } \\
\text { NP-Complete }\end{array}$ & Journal of Computational Biology \\
\hline Gustafsson et al. (2008) & $\begin{array}{l}\text { ALL-TIMES - A European Project on Integrating } \\
\text { Timing Technology }\end{array}$ & $\begin{array}{c}\text { Communications in Computer and } \\
\text { Information Science }\end{array}$ \\
\hline Hogan et al. (2009) & $\begin{array}{l}\text { The Health Impact of a Hearing Disability on Older } \\
\text { People in Australia }\end{array}$ & Journal of Aging and Health \\
\hline Jarimo e Salo (2009) & $\begin{array}{l}\text { Multicriteria Partner Selection in Virtual } \\
\text { Organizations With Transportation Costs and Other } \\
\text { Network Interdependencies }\end{array}$ & $\begin{array}{l}\text { IEEE Transactions on Systems Man } \\
\text { and Cybernetics }\end{array}$ \\
\hline $\begin{array}{c}\text { Kauffman e Sougstad } \\
\text { (2008) }\end{array}$ & $\begin{array}{l}\text { Risk Management of Contract Portfolios in IT } \\
\text { Services: The Profit-at-Risk Approach }\end{array}$ & $\begin{array}{c}\text { Journal of Management } \\
\text { Information Systems }\end{array}$ \\
\hline Ker, Lee e Lin (2008) & $\begin{array}{l}\text { Prediction Models for Transverse Cracking of } \\
\text { Jointed Concrete Pavements Development with } \\
\text { Long-Term Pavement Performance Database }\end{array}$ & Transportation Research Record \\
\hline $\begin{array}{l}\text { Kester, Hultink e } \\
\text { Lauche (2009) }\end{array}$ & Portfolio decision-making genres: A case study & $\begin{array}{c}\text { Journal of Engineering and } \\
\text { Technology Management }\end{array}$ \\
\hline $\begin{array}{l}\text { Kettunen, Salo e Bunn } \\
\text { (2010) }\end{array}$ & $\begin{array}{l}\text { Optimization of Electricity Retailer's Contract } \\
\text { Portfolio Subject to Risk Preferences }\end{array}$ & $\begin{array}{l}\text { IEEE Transactions on Power } \\
\text { Systems }\end{array}$ \\
\hline $\begin{array}{l}\text { Langerak e Hultink } \\
\text { (2008) }\end{array}$ & $\begin{array}{l}\text { The effect of new product development acceleration } \\
\text { approaches on development speed: A case study }\end{array}$ & $\begin{array}{c}\text { Journal of Engineering and } \\
\text { Technology Management }\end{array}$ \\
\hline Lerch et al. (2008) & $\begin{array}{l}\text { Overview of the Mark Twain Lake/Salt River Basin } \\
\text { Conservation Effects Assessment Project }\end{array}$ & $\begin{array}{l}\text { Journal of Soil and Water } \\
\text { Conservation } \\
\end{array}$ \\
\hline
\end{tabular}


Anexo 1. Continuação...

\begin{tabular}{|c|c|c|}
\hline Artigo & $\begin{array}{l}\text { Título } \\
\end{array}$ & Periódico \\
\hline Li et al. (2008) & $\begin{array}{l}\text { Real-time storm detection and weather forecast } \\
\text { activation through data mining and events } \\
\text { processing }\end{array}$ & Arth Science Informatics \\
\hline $\begin{array}{l}\text { Liesio, Mild e Salo } \\
\text { (2008) }\end{array}$ & $\begin{array}{l}\text { Robust portfolio modeling with incomplete cost } \\
\text { information and project interdependencies }\end{array}$ & $\begin{array}{c}\text { European Journal of Operational } \\
\text { Research }\end{array}$ \\
\hline Lin (2008) & $\begin{array}{l}\text { A model using home appliance ownership data to } \\
\text { evaluate recycling policy performance }\end{array}$ & $\begin{array}{l}\text { Resources Conservation and } \\
\text { Recycling }\end{array}$ \\
\hline Lin e Hsu (2010) & $\begin{array}{l}\text { Holistic decision system for human resource } \\
\text { capability identification }\end{array}$ & $\begin{array}{l}\text { Industrial Management \& Data } \\
\text { Systems }\end{array}$ \\
\hline Lin et al. (2009) & $\begin{array}{l}\text { Design for Usability on Supply Chain Management } \\
\text { Systems Implementation }\end{array}$ & $\begin{array}{l}\text { Human Factors and Ergonomics in } \\
\text { Manufacturing }\end{array}$ \\
\hline Lin et al. (2008) & $\begin{array}{l}\text { Pursuing excellence in firm core knowledge through } \\
\text { intelligent group decision support system }\end{array}$ & $\begin{array}{c}\text { Industrial Management \& Data } \\
\text { Systems }\end{array}$ \\
\hline $\begin{array}{l}\text { Lin, Huang e Shaw } \\
(2010)\end{array}$ & $\begin{array}{l}\text { Applying Water Quality Modeling to Regulating } \\
\text { Land Development in a Watershed }\end{array}$ & Water Resources Management \\
\hline Lin, Wen e Tsai (2010) & $\begin{array}{l}\text { Applying decision-making tools to national e-waste } \\
\text { recycling policy: An example of Analytic Hierarchy } \\
\text { Process }\end{array}$ & Waste Management \\
\hline $\begin{array}{l}\text { Lindstedt, Liesio e Salo } \\
\text { (2008) }\end{array}$ & $\begin{array}{l}\text { Participatory development of a strategic product } \\
\text { portfolio in a telecommunication company }\end{array}$ & $\begin{array}{c}\text { International Journal of Technology } \\
\text { Management }\end{array}$ \\
\hline Mild e Salo (2009) & $\begin{array}{l}\text { Combining a Multiattribute Value Function with an } \\
\text { Optimization Model: An Application to Dynamic } \\
\text { Resource Allocation for Infrastructure Maintenance }\end{array}$ & Decision Analysis \\
\hline $\begin{array}{l}\text { Miller, Chomcynova e } \\
\text { Beck (2009) }\end{array}$ & $\begin{array}{l}\text { Predicting Teenage Beliefs Concerning the Harm } \\
\text { Alcohol and Cannabis Use may do in Eight } \\
\text { European Countries }\end{array}$ & Journal of Substance Use \\
\hline Peng et al. (2009) & $\begin{array}{l}\text { Testing of Holes in Concrete Using Gamma } \\
\text { Radiography }\end{array}$ & Materials Evaluation \\
\hline $\begin{array}{l}\text { Sargaonkar, Gupta e } \\
\text { Devotta (2008) }\end{array}$ & $\begin{array}{l}\text { Evaluation of Monitoring Sites for Protection of } \\
\text { Groundwater in an Urban Area }\end{array}$ & Water Environment Research \\
\hline $\begin{array}{l}\text { Singh, Einstadter e } \\
\text { Lawrence (2010) }\end{array}$ & $\begin{array}{l}\text { A structured women's preventive health clinic for } \\
\text { residents: a quality improvement project designed } \\
\text { to meet training needs and improve cervical cancer } \\
\text { screening rates }\end{array}$ & Quality \& Safety in Health Car \\
\hline $\begin{array}{l}\text { Van Bekkum, Pennings } \\
\text { e Smit (2009) }\end{array}$ & $\begin{array}{l}\text { A real options perspective on } R \& D \text { portfolio } \\
\text { diversification }\end{array}$ & Research Policy \\
\hline Varma et al. (2008) & $\begin{array}{l}\text { A framework for addressing stochastic and } \\
\text { combinatorial aspects of scheduling and resource } \\
\text { allocation in pharmaceutical R\&D pipelines }\end{array}$ & $\begin{array}{l}\text { Computers \& Chemical } \\
\text { Engineering }\end{array}$ \\
\hline $\begin{array}{l}\text { Vijay, Sargaonkar e } \\
\text { Gupta (2009) }\end{array}$ & $\begin{array}{l}\text { A hydrodynamic approach to address Yamuna } \\
\text { riverbed development in Delhi }\end{array}$ & $\begin{array}{l}\text { Canadian Journal of Civil } \\
\text { Engineering } \\
\end{array}$ \\
\hline
\end{tabular}

